\title{
Follow the bouncing ball: global results on tracking and state estimation with impacts
}

\author{
F. Forni, A.R. Teel, L. Zaccarian
}

\begin{abstract}
In this paper we formulate tracking and state-estimation problems of a translating mass in a polyhedral billiard as a stabilization problem for a suitable set. Due to the discontinuous trajectories arising from the impacts, we use hybrid systems stability analysis tools to establish the results. Using a novel concept of mirrored images of the target mass we prove that 1) a tracking control algorithm, and 2) an observer algorithm guarantee global exponential stability results for specific classes of polyhedral billiards, including rectangles. Moreover, we combine these two algorithms within dynamic controllers that guarantee global output feedback tracking. The results are illustrated via simulations.
\end{abstract}

\section{INTRODUCTION}

Control and state estimation of dynamical systems subject to impacts are relevant problems in several application areas, often related to the robotics field [2], and impacts play a key role in several studies including hopping robots (see, e.g., [27]), walking robots (see, e.g., [20]) and juggling robots (see, e.g., [23]). Several Lyapunov-based solutions to the stabilization and tracking problem of systems with impacts have been proposed in the past decade [3], [14], [30], and several studies have been developed for the dual state-estimation problem [18], [17], [10]. Some of them address the problem via the larger class of complementarity Lagrangian systems. These systems are a specific class of hybrid systems where the state is subject to a jump or re-initialization rule whenever a unilateral constraint is reached (see [13] for a survey and [19] which also improves the results in [1], [4]). Several additional recent techniques addressing tracking control with impacts both from a theoretical and an experimental viewpoint are provided in the works [21], [22], [15], [16], [28] and references therein. See [19] for a more detailed overview. Tracking control in billiards is a representative example of the control problem discussed above whenever the control action is allowed to act during the motion (like, e.g, in walking robots) and the impacts correspond to jumps in the state occurring whenever the trajectory reaches a constraint. In this context, a number of results have been produced, which rely on the model first proposed in [30]. These are nicely summarized in [9], where the technique is used for tracking a reference mass moving in an elliptical billiard (circular ones were considered in [16]). The parallel problem of tracking trajectories while restricting the control action at the impact times is addressed in [23], [24] and references therein.

The problem statement in this paper is motivated by [9], [16] where Lyapunov-based tracking control is designed for a mass moving on a billiard. We cast this problem within the framework of [12], [11] for hybrid dynamical systems and we propose a novel control strategy inducing global decrease of a suitable Lyapunov function. Preliminary results of this paper appeared in [7], [8]. This type of approach is new in the area as most of the existing Lyapunov-based results treat the impacts as events which locally increase the Lyapunov function and resort to weak stability concepts. (Notable exceptions can be found in [21] and [18].) In this paper, instead, we provide a Lyapunov function that does not increase at impacts and that can be used to establish stable asymptotic tracking with uniform global exponential convergence for several types of polyhedral billiards. Such global results are rare in the literature. The hybrid framework of [12], [11] greatly facilitates the analysis.

A.R. Teel is with the ECE Department, University of California, Santa Barbara, CA 93106-9560. His research supported in part by AFOSR grant FA9550-09-1-0203 and NSF grants ECCS-0925637 and CNS-0720842.

L. Zaccarian is with CNRS, LAAS; Université de Toulouse, F-31077, Toulouse, France and is supported by HYCON2 Network of Excellence "Highly-Complex and Networked Control Systems", grant agreement 257462.

F. Forni is with the EECS Department, University of Liege, Belgium. This paper presents research results of the Belgian Network DYSCO (Dynamical Systems, Control, and Optimization), funded by the Interuniversity Attraction Poles Programme, initiated by the Belgian State, Science Policy Office. The scientific responsibility rests with its author(s). 


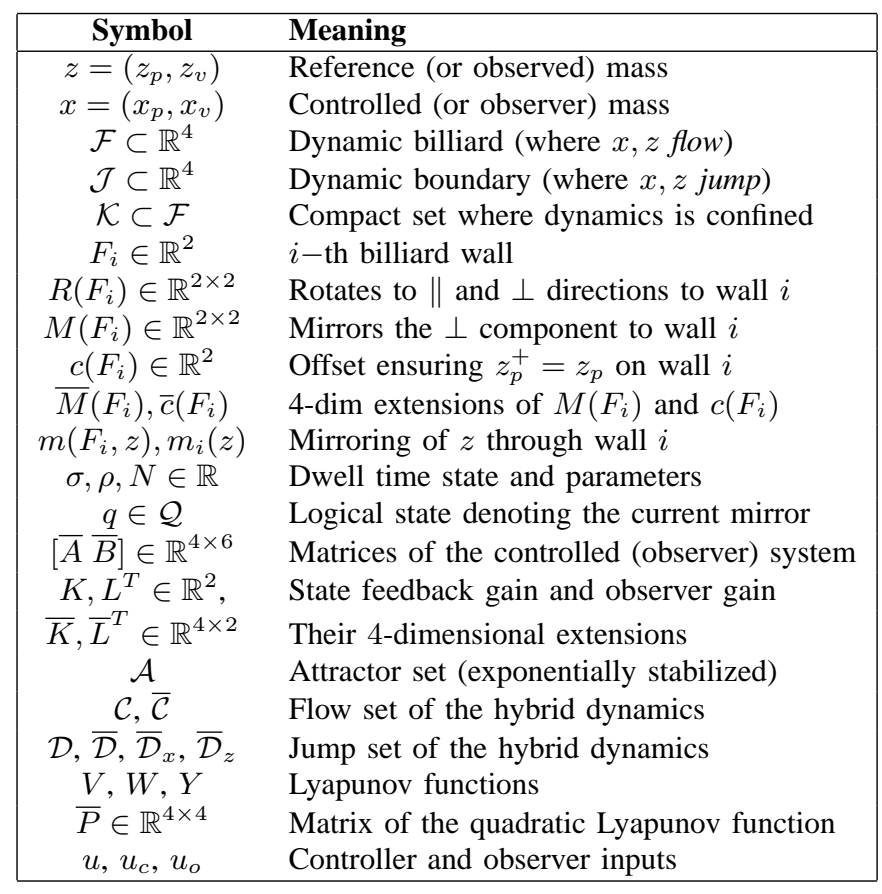

Table I

TABLE OF NOTATION.

We propose to compute the tracking/observer error based on a suitable selection of the mirrored image of the reference through the billiard boundaries. It is notable that a so-called "mirror algorithm" is proposed in [5] to solve the juggling problem. This has little similarity with our approach as the "mirror" is used there at all times to make the actuator track a mirror image of the ball trajectory (which is regulated by acting at impacts), whereas here it is used to prevent the algorithms (which act during continuous motion) from getting confused by the impacts (see, for example, the explanation in Remark 4). The tracking and observer algorithms proposed here share several similarities (duality), since both require a selection of the stabilizing control/observer gains based on classical linear system theory (the selection must guarantee that a specific matrix is Hurwitz, thus allowing for arbitrarily small gains), they both satisfy the set of constraints enforced by the boundaries of the billiard, and satisfy also a separation principle which leads to the construction of a global output feedback tracking algorithm. To the best of the knowledge of the authors, global results for tracking, state estimation and output feedback tracking with impacts are new in literature, with the exception of [18] which presents a global observer for linear mechanical systems impacting on a single boundary.

The paper uses the hybrid system framework summarized in [11]. In particular, we use $\dot{x} \in F(x)$ to characterize the continuous dynamics of the system, which may occur when the state $x$ belongs to the flow set $\mathcal{C} \subset \mathbb{R}^{n}$. The impulsive dynamics at impacts is characterized by the update inclusion $x^{+} \in G(x)$, which may occur when $x$ belongs to the jump set $\mathcal{D} \in \mathbb{R}^{n}$. The concept of solution, several results on stability, invariance principles and robustness for hybrid systems, can be found in [11], [12], [25], [26]. The paper is structured as follows. In Section II we introduce the notion of polyhedral billiards and describe a hybrid model. In Sections III and IV, we present global results on tracking and state estimation developed for billiards with one boundary and, under appropriate assumptions, in Section V we extend these results to billiards with multiple walls. The two approaches are combined in Section VI to design an output feedback controller.

Notation: The Euclidean norm of a vector is denoted by $|\cdot|$. For any given set $\mathcal{A}$, the quantity $|x|_{\mathcal{A}}$ denotes the distance of $x$ to $\mathcal{A}$, that is, $|x|_{\mathcal{A}}:=\inf _{a \in \mathcal{A}}|x-a|$. Given a matrix $P=P^{T}>0,|x|_{P}:=\sqrt{x^{T} P x}$. Given two matrices $A, B$, then $A \otimes B$ denotes their Kronecker product. A matrix is Hurwitz if all its eigenvalues have negative real part. Given a function $(x, y) \mapsto f(x, y)$, then $\nabla_{x} f(x, y):=\left[\frac{\partial f(x, y)}{\partial x}\right]^{T}$. For any given vectors $x$ and $y,\langle x, y\rangle=x^{T} y$. For $n \in \mathbb{N}, I_{n}$ denotes the 
identity matrix $I \in \mathbb{R}^{n \times n}$. Given two sets $\mathcal{K}, \mathcal{F}, \mathcal{K} \subset \mathcal{F}$ means that every element of $\mathcal{K}$ is also an element of $\mathcal{F}$. Table I reports a selection of the main symbols used in the paper.

\section{THE HYBRID DYNAMICS}

Consider two translating masses $\mathcal{Z}$ and $\mathcal{X}$ moving within a convex polyhedral region and subject to impacts. In the typical scenario, $\mathcal{Z}$ is the reference system and $\mathcal{X}$ is the controlled/observer system and we aim to make the state vector $x$ of $\mathcal{X}$ track or estimate the state vector $z$ of $\mathcal{Z}$. For simplicity, we decompose each state vector $s \in \mathbb{R}^{4}$ into $s_{p} \in \mathbb{R}^{2}$ and $s_{v} \in \mathbb{R}^{2}$, denoting respectively position and velocity subvectors, and we call billiard the polyhedral region $\mathcal{F}$ constraining the motion of the masses, to emphasize the fact that the dynamics of $\mathcal{Z}$ and $\mathcal{X}$ resemble the behavior of two balls moving on a billiard and impacting on its boundary. A billiard is defined by

$$
\mathcal{F}:=\left\{s \in \mathbb{R}^{4} \mid \forall i \in \mathcal{I},\left\langle F_{i}, s_{p}-s_{\circ}\right\rangle \leq 1\right\}
$$

where $r$ is the number of billiard walls, $F_{i} \in \mathbb{R}^{2}, i \in \mathcal{I}=\{1, \ldots, r\} \subset \mathbb{N}$, fix the shape of the billiard and $s_{\circ}$ fixes its location in the plane. The dynamic boundary $\mathcal{J}$ of the billiard is

$$
\mathcal{J}:=\left\{s \in \mathcal{F} \mid \exists i \in \mathcal{I},\left\langle F_{i}, s_{p}-s_{\circ}\right\rangle=1,\left\langle F_{i}, s_{v}\right\rangle \geq 0\right\}
$$

where, by $\left\langle F_{i}, s_{v}\right\rangle \geq 0, s$ belongs to $\mathcal{J}$ when the velocity subvector $s_{v}$ triggers an impact. Figure 1 represents the case of a one-wall billiard with $s_{\circ}=0$.

Postponing the description of the controlled/observer system structure to the next sections, the continuous motion of the reference system is characterized by the following equations

$$
\mathcal{Z}:\left\{\begin{array}{lll}
\dot{z}_{p} & =z_{v} \\
\dot{z}_{v} & \in & \alpha(z)
\end{array}\right.
$$

where $\alpha: \mathbb{R}^{4} \rightrightarrows \mathbb{R}^{2}$ is a set-valued mapping that satisfies mild regularity conditions (which are made precise later, in Assumption 1). A special case covered here is when $\alpha$ is replaced by a continuous function defined on $\mathcal{F}$. We allow for set-valued accelerations for the reference variable $z_{p}$ in order to allow for nonunique trajectories of $z$. While the acceleration is not assumed to be unique, the selected acceleration at each time is assumed to be known by the control/estimation algorithm. When the acceleration is not assumed to be known but a bound on the acceleration is known, its effect typically can be mitigated using high feedback or observer gains.

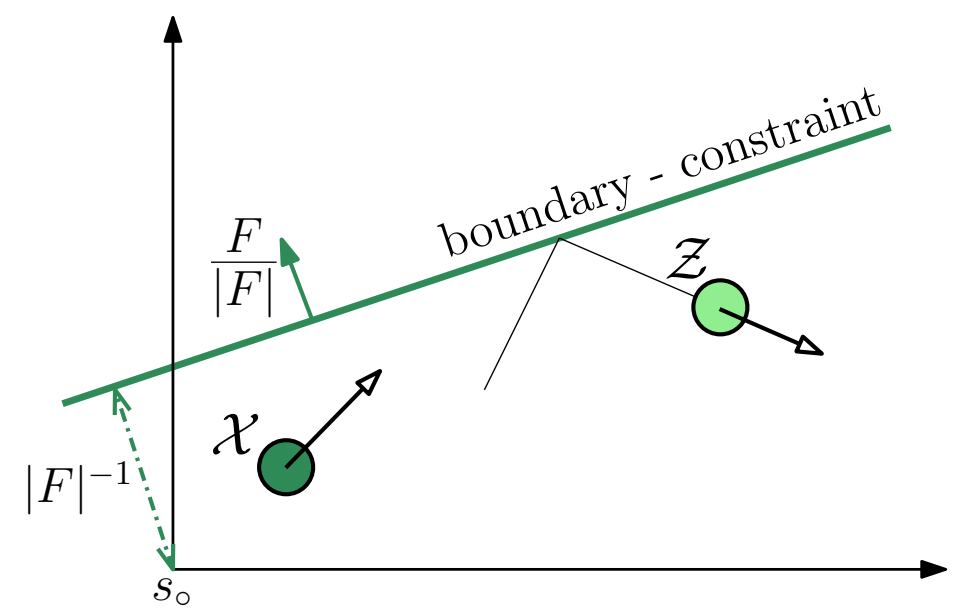

Figure 1. Two translating masses $\mathcal{Z}$ and $\mathcal{X}$ in a one-wall billiard with $s_{\circ}=0$.

An impact on the wall $F$ occurs when the position subvector $z_{p}$ satisfies $\left\langle F, z_{p}-s_{\circ}\right\rangle=1$ and the velocity subvector $z_{v}$ pierces (or is parallel to) the wall, ${ }^{1}$ that is, $\left\langle F, z_{v}\right\rangle \geq 0$. The position does not

\footnotetext{
${ }^{1}$ Our model permits trajectories that graze a wall and corresponding jumps that do not change the state; however, the number of such jumps is limited by an average dwell-time mechanism that is introduced into the model later, in (7).
} 
change at impacts, that is, $z_{p}^{+}=z_{p}$, while the velocity is reflected (reset) in a direction that is determined by the velocity $z_{v}$ before the impact and the wall orientation $F$, given by $z_{v}^{+}=M(F) z_{v}$, where

$$
\begin{aligned}
M(F) & :=R(F)^{T} \operatorname{diag}(1,-1) R(F), \\
R(F) & :=\frac{1}{|F|}\left[\begin{array}{c}
F^{T} J \\
F^{T}
\end{array}\right], \quad J:=\left[\begin{array}{rr}
0 & -1 \\
1 & 0
\end{array}\right]
\end{aligned}
$$

with $|F|=\sqrt{F^{T} F}$. In fact, $M(F)$ inverts only the component of $z_{v}$ normal to the wall $F$, by combining the matrix $\left[\begin{array}{cc}1 & 0 \\ 0 & -1\end{array}\right]$ with the the rotation matrix $R(F)$ that transforms the Cartesian components $z_{v}$ of the velocity into the coordinate system $\left\{\left[\begin{array}{cc}0 & 1 \\ -1 & 0\end{array}\right] \frac{F}{|F|}, \frac{F}{|F|}\right\}$ whose components correspond to the tangential and normal directions to the wall, respectively. Thus, the impact dynamics can be compactly written as

$$
\begin{aligned}
& z^{+} \in \bigcup_{i \in \mathcal{M}(z)}\left[\begin{array}{c}
z_{p} \\
M\left(F_{i}\right) z_{v}
\end{array}\right]=\bigcup_{i \in \mathcal{M}(z)} m\left(F_{i}, z\right) \\
& \mathcal{M}(z):=\left\{i \in \mathcal{I} \mid\left\langle F_{i}, z_{p}-s_{\circ}\right\rangle=1,\left\langle F_{i}, z_{v}\right\rangle \geq 0\right\}
\end{aligned}
$$

where, for each vector $F_{i}$ (associated to wall $i$ ),

$$
\begin{aligned}
c(F) & :=F \cdot 2\left(1+F^{T} s_{\circ}\right) /|F|^{2} \\
\bar{M}(F) & :=\operatorname{diag}(M(F), M(F))=I_{2} \otimes M(F) \\
\bar{c}(F) & :=\left[\begin{array}{ll}
c(F)^{T} & 0_{1 \times 2}
\end{array}\right]^{T}=\left[\begin{array}{l}
1 \\
0
\end{array}\right] \otimes c(F) \\
m(F, z) & :=\bar{M}(F) z+\bar{c}(F),
\end{aligned}
$$

and for convenience of notation, we use $M(0)=I$ and $c(0)=0$. The union for $i \in \mathcal{M}(z)$ in (5) is motivated by the fact that the reflection of the velocity vector is not unique when $\mathcal{Z}$ impacts a point shared by two walls $F_{i}$ and $F_{j}, i, j \in \mathcal{I}$, so that $\mathcal{M}$ is not a singleton (this point can be intuitively visualized as a billiard "corner", where two walls intersect). The next claim establishes some useful relations.

Claim 1: Given the quantities in (6),

(i) $M(F) M(F)=M(F)^{T} M(F)=I$;

(ii) $(M(F)+I) c(F)=0$;

(iii) $F^{T} M(F) s=-F^{T} s$, for all $s \in \mathbb{R}^{2}$;

(iv) $s=M(F) s+c(F)$ iff $\left\langle F, s-s_{\circ}\right\rangle=1$, for all $s \in \mathbb{R}^{2}$;

(v) $m(F, m(F, s))=s$, for all $s \in \mathbb{R}^{4}$;

(vi) $|\bar{M}(F) s|=|s|$, for all $s \in \mathbb{R}^{4}$.

Proof: (i) $M(F) M(F)=R(F)^{T}\left[\begin{array}{cc}1 & 0 \\ 0 & -1\end{array}\right]\left[\begin{array}{cc}1 & 0 \\ 0 & -1\end{array}\right] R(F)=R(F)^{T} R(F)=I$, and $M(F)=M(F)^{T}$. (ii) $(M(F)+I) c(F)=\frac{2}{|F|^{2}}\left[J^{T} F \star\right]\left[\begin{array}{cc}2 & 0 \\ 0 & 0\end{array}\right]\left[\begin{array}{c}F^{T} J \\ \star\end{array}\right] F\left(1+F^{T} s_{\circ}\right) /|F|^{2}=0$ by $F^{T} J F=0$, where $\star$ indicates quantities multiplied by zero. (iii) $F^{T} M(F) s=\frac{F^{T}}{|F|^{2}}\left[\begin{array}{ll}J^{T} & F\end{array}\right]\left[\begin{array}{cc}1 & 0 \\ 0 & -1\end{array}\right]\left[\begin{array}{c}\stackrel{\star}{F^{T}}\end{array}\right] s=\left[\begin{array}{ll}0 & 1\end{array}\right]\left[\begin{array}{c}\star \\ -F^{T}\end{array}\right] s=-F^{T} s$. (iv) Consider $\left\langle F, s-s_{\circ}\right\rangle=\alpha$ then $(I-M(F)) s=\frac{1}{|F|^{2}}[\star F]\left[\begin{array}{ll}0 & 0 \\ 0 & 2\end{array}\right]\left[\begin{array}{c}\star \\ F^{T}\end{array}\right] s=\frac{2 \alpha F}{|F|^{2}}+2 F F^{T} s_{\circ} /|F|^{2}=c(F)$ when $\alpha=1$. (v) From the definitions in (6), for the velocity vector we have $s_{v}=M(F) M(F) s_{v}$ by (i) above, while for the position vector we have $M(F)\left(M(F) s_{p}+c(F)\right)+c(F)=s_{p}+(M(F)+I) c(F)=s_{p}$ by (i) and (ii) above. (vi) We have to prove that $|M(F) s|=|s|$ for each $s \in \mathbb{R}^{2}$. Indeed, $|M(F) s|=$ $\sqrt{s^{T} M(F)^{T} M(F) s}=\sqrt{s^{T} s}=|s|$, by (i) above.

For reasons of control design, we restrict the motion of $\mathcal{Z}$ within a compact set $\mathcal{K}$ that prevents the state $z$ from reaching any billiard corner.

Assumption 1: For the compact set $\mathcal{K} \subset \mathcal{F}$, if $z \in \mathcal{J} \cap \mathcal{K}$ then $\mathcal{M}(z)$ is a singleton. Moreover, $\alpha$ in (3) is outer semicontinuous, ${ }^{2}$ locally bounded, and $\alpha(z)$ is nonempty and convex $\forall z \in \mathcal{F}$.

\footnotetext{
${ }^{2}$ Namely, for each converging sequence $\left(y_{i}, z_{i}\right)$ with $y_{i} \in \alpha\left(z_{i}\right)$ for all $i$, we have $y \in \alpha(z)$ where $(y, z)=\lim { }_{i \rightarrow \infty}\left(y_{i}, z_{i}\right)$.
} 
Moreover, to rule out solutions that jump infinitely many times and never evolve continuously, which can occur when $\mathcal{Z}$ impacts a wall with a velocity that is either zero or tangent to the wall ${ }^{3}$ we augment the plant with an average dwell-time automaton [6], [11, eq. (S3), (S4)]. In particular, let $N$ be a positive integer and $\rho>0$, we add the dynamics

$$
\begin{aligned}
\dot{\sigma} & \in[0, \rho] & & \sigma \in[0, N] \\
\sigma^{+} & =\sigma-1 & & \sigma \in[1, N] .
\end{aligned}
$$

The hybrid dynamics arising from the continuous evolution (flow) of $\mathcal{Z}$ according to (3), (7a) and the discrete evolution (jump) of $\mathcal{Z}$ according to (5), (7b) can be represented using the hybrid formalism in [11], selecting the flow set (where the system can flow) as $(z, \sigma) \in \mathcal{K} \times[0, N]$ and the jump set (where the system can jump) as $\sigma \in[1, N]$ and $z \in \mathcal{J} \cap \mathcal{K}$.

Remark 1: For a hybrid system having state in $\mathbb{R}^{n}$, sequences of flows and jumps which possibly characterize solutions [12] to a hybrid system are typically denoted by functions $\xi: \operatorname{dom} \xi \rightarrow \mathbb{R}^{n}$, where $\operatorname{dom} \xi$ is a subset of $\mathbb{R}_{\geq 0} \times \mathbb{N}$ called hybrid time domain [12], given by the union of infinitely many intervals of the form $\left[t_{j}, t_{j+1}\right] \times\{j\}$ where $0=t_{0} \leq t_{1} \leq t_{2} \leq \ldots$, or of finitely many such intervals, with the last one possibly of the form $\left[t_{j}, t_{j+1}\right] \times\{j\},\left[t_{j}, t_{j+1}\right) \times\{j\}$, or $\left[t_{j}, \infty\right) \times\{j\}$. According to [6], the dwell-time automaton (7) guarantees that any solution $\xi$ has a hybrid time domain such that for any pair $(t, j),(s, i) \in \operatorname{dom} \xi$ satisfying $t+j \geq s+i$, we have $j-i \leq \rho(t-s)+N$, which clearly imposes an upper bound on the total number of jumps that occur between the two hybrid times depending on the amount of flow elapsed between them. As a consequence, no Zeno solution can occur. Note that larger values of $\rho$ and $N$ will impose less stringent bounds on the average dwell-time constraint.

Remark 2: We emphasize that the average dwell time parameters $(\rho, N)$ are not used in the control and observer design, which implies that our global exponential properties hold for any selection of these parameters, that is, for any arbitrarily fast impact occurrence. Therefore, the only effect of the automaton (7) is to remove defective Zeno solutions from our analysis.

\section{STATE FEEDBACK TRACKING: SINGLE WALL}

We first present our solution for impacts occurring on a single wall. The proposed approach will be used in the next sections when addressing multiple walls because we characterize a wall with an arbitrary orientation. Nevertheless, much intuition can be gained by focusing on the special case of the wall being the horizontal axis, namely the set $s_{2}=0$ which, using $s_{\circ}=\left[\begin{array}{l}0 \\ 1\end{array}\right]$ gives $F=\left[\begin{array}{c}0 \\ -1\end{array}\right]$. See Remark 4 for a few observations regarding this special case.

\section{A. Controlled system}

Consider a controlled system $\mathcal{X}$ which is controlled only during the continuous-time evolution and consider the goal of finding a control input $u$ for that system that guarantees asymptotic convergence of the position $x_{p}$ to the position $z_{p}$ of the reference system $\mathcal{Z}$. The dynamics of the controlled system $\mathcal{X}$ resembles that of $\mathcal{Z}$ as follows:

$$
\mathcal{X}: \dot{x}=\bar{A} x+\bar{B}(\phi(x)+u), \quad x \in \mathcal{F}
$$

where $\bar{A}=\left[\begin{array}{ll}0 & I \\ 0 & 0\end{array}\right] \in \mathbb{R}^{4 \times 4}, \bar{B}=\left[\begin{array}{l}0 \\ I\end{array}\right] \in \mathbb{R}^{4 \times 2}, \phi: \mathbb{R}^{4} \rightarrow \mathbb{R}^{2}$ is a continuous function representing possible nonlinear terms characterizing the dynamics of $\mathcal{X}$, and $u$ is the control input; the impact dynamics is given by

$$
\mathcal{X}: x^{+} \in \bigcup_{i \in \mathcal{M}(x)} m\left(F_{i}, x\right) \quad x \in \mathcal{J} .
$$

The flow dynamics of the complete system is given by (8), (3) and (7a) when $(x, z, \sigma) \in \mathcal{C}$,

$$
\mathcal{C}:=\mathcal{F} \times \mathcal{K} \times[0, N]
$$

${ }^{3}$ By (iii) of Claim $1,\left\langle F, s_{v}\right\rangle>0$ if and only if $\left\langle F, s_{v}^{+}\right\rangle\left\langle 0\right.$, thus $s \in \mathcal{J}$ and $s^{+} \in \mathcal{J}$ may occur only when $\left\langle F, s_{v}\right\rangle=0$. 
while the jump dynamics is given by (9), $z^{+}=z$ and (7b) when $(x, z, \sigma) \in \mathcal{D}_{x}$, and by $x^{+}=x$, (5) and (7b) when $(x, z, \sigma) \in \mathcal{D}_{z}$, where

$$
\begin{aligned}
\mathcal{D}_{x} & :=\mathcal{J} \times \mathcal{K} \times[1, N] \\
\mathcal{D}_{z} & :=\mathcal{F} \times(\mathcal{J} \cap \mathcal{K}) \times[1, N], \\
\mathcal{D} & :=\mathcal{D}_{x} \cup \mathcal{D}_{z} .
\end{aligned}
$$

\section{B. Control algorithm for a single wall billiard}

The tracking problem may be addressed by an input $u$ that enforces asymptotic convergence to zero of the $x-z$ dynamics by asymptotically stabilizing the set $\mathcal{A}_{\circ}=\{(x, z) \mid x=z\}$ in the absence of impacts. But in the presence of impacts, classical algorithms may fail to guarantee stability and convergence, as shown in the following example.

Example 1: In Figure 2 the horizontal motion of the two masses $\mathcal{Z}$ and $\mathcal{X}$ is constrained on the left by a wall placed at 0 . The continuous dynamics is given by $\dot{z}=\left[\begin{array}{ll}0 & 1 \\ 0 & 0\end{array}\right] z+\left[\begin{array}{c}0 \\ -\mu\end{array}\right]$ where $\mu>0$ is a constant external force, and $\dot{x}=\left[\begin{array}{ll}0 & 1 \\ 0 & 0\end{array}\right] x+\left[\begin{array}{l}0 \\ 1\end{array}\right] u$ where the input $u=-\mu+[-4-4](x-z)$ guarantees that the matrix $A=\left[\begin{array}{cc}0 & 1 \\ -4 & -4\end{array}\right]$ of the error dynamics $\dot{x}-\dot{z}=A(x-z)$ is Hurwitz.
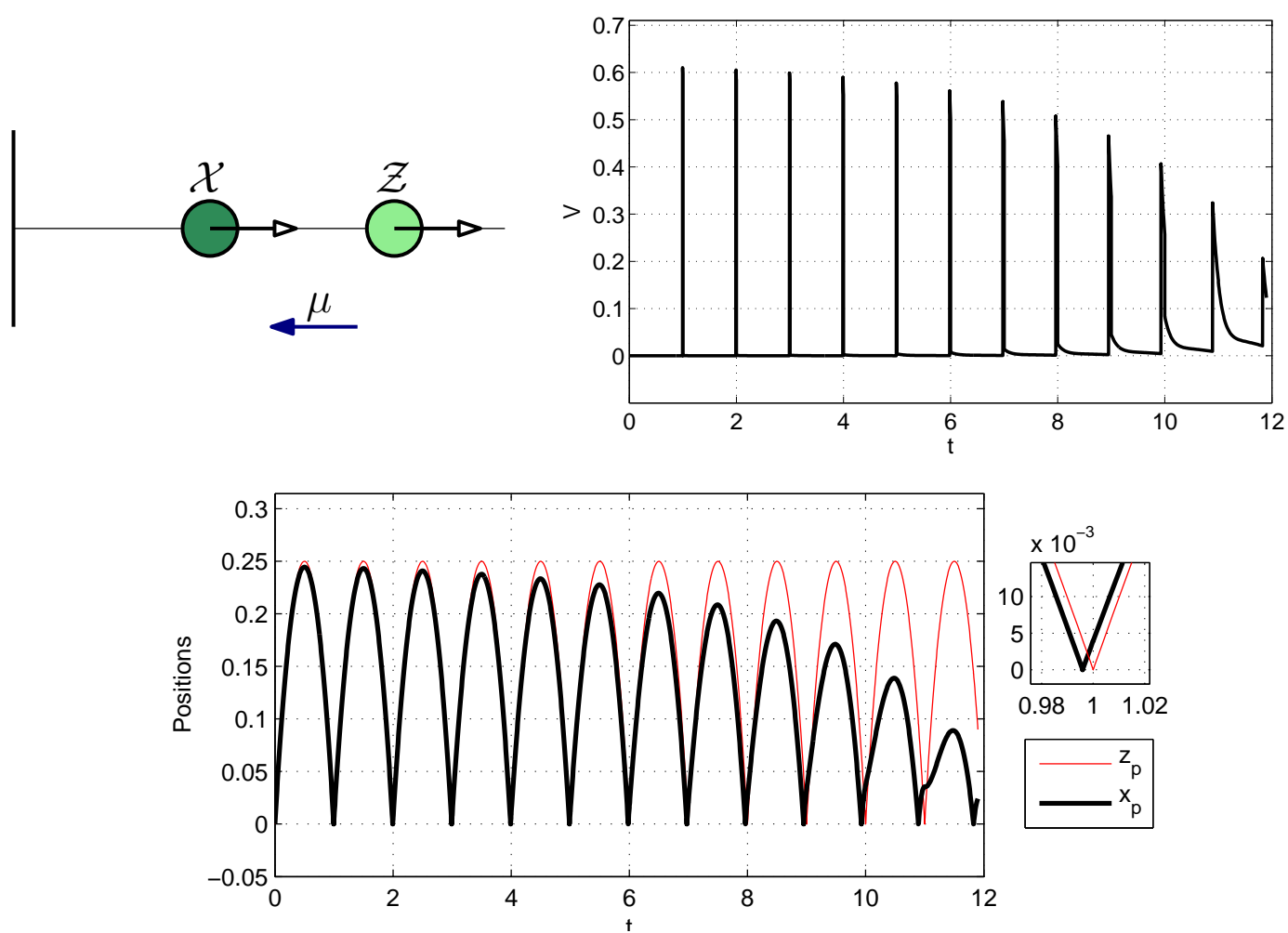

Figure 2. Example 1: Sketch of the two masses (top-left); time evolution of the function $V(x, z)=(x-z)^{T} P(x-z)$ where $P=P^{T}>0$ satisfies $A^{T} P+P A \leq-I$ (top-right); time evolution of the positions of $\mathcal{X}$ and $\mathcal{Z}$ (bottom).

Given $z_{0}=[0 v]^{T}$ and $x_{0}=z_{0}+\varepsilon$, with $\varepsilon \in \mathbb{R}^{2}$ typically small, for a specific set of initial mismatches $\varepsilon$ defined next, the cyclic behavior of the two masses can be qualitatively characterized as a sequence of a continuous motion (where the two masses reverse their direction under the effect of the force $\mu$ ), followed by the impact of $\mathcal{X}$ to the wall, then by the impact of $\mathcal{Z}$, from which this sequence repeats. The mismatch $x-z$ at the $k$ th impact of $\mathcal{Z}$ is given approximately, with increasing accuracy for smaller values of $\varepsilon$, by

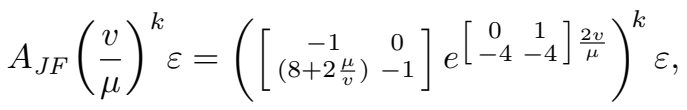

where the matrix $A_{J F}\left(\frac{v}{\mu}\right)$ (whose deduction is given below) presents an unstable eigenvalue when the ratio $\frac{v}{\mu}$ is smaller than 0.613 . For example, given $v=1$ and $\mu=2$, the value of the unstable eigenvalue is 1.34 
and the corresponding eigenvector is $\zeta=[0.0773-0.997]^{T}$, thus picking $\varepsilon=\lambda \zeta$, with $0<\lambda \ll 1$, we have that the error $e=x-z$ immediately after the $k$ th impact of $\mathcal{Z}$ is given by $1.34^{k} \varepsilon$, i.e. impacts destabilize the system. Note that the other eigenvalue of $A_{J F}$ has norm less than one, therefore the unstable behavior would appear also for an initial mismatch $\varepsilon$ near $\lambda \zeta, 0<\lambda \ll 1$.

Using the notation of hybrid time domains introduced in Remark $1, \mu=2$ and $v=1$, consider $e(0,0)=x_{0}-z_{0}=\varepsilon \simeq \lambda \zeta, 0<\lambda \ll 1$. For $v>0$ and $\lambda$ small, the time spent by $\mathcal{Z}$ and $\mathcal{X}$ to go back to zero is given approximately by $\tau_{1}:=\frac{2 v}{\mu}$, and for $t \in\left[0, \tau_{1}\right]$ the time evolution of the error is given by $e(t, 0)=\exp (A t) e(0,0)$. Thus, defining $A_{F}\left(\frac{v}{\mu}\right):=\exp \left(A \frac{2 v}{\mu}\right)$, immediately before the first impact we have $e\left(\tau_{1}, 0\right):=A_{F}\left(\frac{v}{\mu}\right) e(0,0)=\left[\begin{array}{cc}0.4060 & 0.1353 \\ -0.5413 & -0.1353\end{array}\right] e(0,0)$, from which we can also infer that $\mathcal{X}$ impacts first since for $e(0,0) \simeq \lambda \zeta, e\left(\tau_{1}, 0\right) \simeq \lambda[-0.10350 .0931]^{T}$, that is, $x_{p}\left(\tau_{1}, 0\right)<z_{p}\left(\tau_{1}, 0\right)$. Consider now the interval of time between the impact of $\mathcal{X}$ and the impact of $\mathcal{Z}$ which is given approximately by $\tau_{2}:=-\frac{e_{p}\left(\tau_{1}, 0\right)}{v}$. When $\mathcal{X}$ impacts, the position does not change, $x_{p}\left(\tau_{1}, 1\right)=x_{p}\left(\tau_{1}, 0\right)=0$, while the velocity resets from $x_{v}\left(\tau_{1}, 0\right)=e_{v}\left(\tau_{1}, 0\right)+z_{v}\left(\tau_{1}, 0\right)=e_{v}\left(\tau_{1}, 0\right)+\left(-v+\mu \tau_{2}\right)=e_{v}\left(\tau_{1}, 0\right)-\left(v+\frac{\mu}{v} e_{p}\left(\tau_{1}, 0\right)\right)$ to $x_{v}\left(\tau_{1}, 1\right)=v+\frac{\mu}{v} e_{p}\left(\tau_{1}, 0\right)-e_{v}\left(\tau_{1}, 0\right)$, from which we have $e\left(\tau_{1}, 1\right) \simeq\left[\begin{array}{c}e_{p}\left(\tau_{1}, 0\right), \\ 2 v+2 \frac{\mu}{v} e_{p}\left(\tau_{1}, 0\right)-e_{v}\left(\tau_{1}, 0\right)\end{array}\right]$. The input between the two impacts can be approximated by $u=-\mu+[-4-4] e\left(\tau_{1}, 1\right) \simeq-\mu-8 v$, from which the error dynamics between the impacts is approximatively given by $\dot{e}_{p}=e_{v}$ and $\dot{e}_{v}=-8 v$. Thus, by integration, at time $\left(\tau_{1}+\tau_{2}, 1\right)$ (immediately before the impact of $\mathcal{Z}$ ), we have $e_{v}\left(\tau_{1}+\tau_{2}, 1\right)=$ $e_{v}\left(\tau_{1}, 1\right)-8 v \tau_{2}=2 v+2 \frac{\mu}{v} e_{p}\left(\tau_{1}, 0\right)-e_{v}\left(\tau_{1}, 0\right)+8 v \frac{e_{p}\left(\tau_{1}, 0\right)}{v} \simeq 2 v-e_{v}\left(\tau_{1}, 0\right)+\left(8+2 \frac{\mu}{v}\right) e_{p}\left(\tau_{1}, 0\right)$ and $e_{p}\left(\tau_{1}+\right.$ $\left.\tau_{2}, 1\right)=e_{p}\left(\tau_{1}, 1\right)+e_{v}\left(\tau_{1}, 1\right) \tau_{2}-4 v \tau_{2}^{2} \simeq-e_{p}\left(\tau_{1}, 0\right)$, from which $e\left(\tau_{1}+\tau_{2}, 1\right)=\left[\begin{array}{c}-e_{p}\left(\tau_{1}, 0\right) \\ 2 v-e_{v}\left(\tau_{1}, 0\right)+\left(8+2 \frac{\mu}{v}\right) e_{p}\left(\tau_{1}, 0\right)\end{array}\right]$. From here, $\mathcal{Z}$ impacts and reverses its speed, from which the mismatch is given by $e\left(\tau_{1}+\tau_{2}, 2\right)=$ $\left[\begin{array}{c}e_{p}\left(\tau_{1}+\tau_{2}, 1\right) \\ -2 v+e_{v}\left(\tau_{1}+\tau_{2}, 1\right)\end{array}\right]=\left[\begin{array}{c}-e_{p}\left(\tau_{1}, 0\right) \\ -e_{v}\left(\tau_{1}, 0\right)+\left(8+2 \frac{\mu}{v}\right) e_{p}\left(\tau_{1}, 0\right)\end{array}\right]=A_{J}\left(\frac{\mu}{v}\right) e\left(\tau_{1}, 0\right)$, where $A_{J}\left(\frac{\mu}{v}\right):=\left[\begin{array}{cc}-1 & 0 \\ 8+2 \frac{\mu}{v} & -1\end{array}\right]$. Finally, after both impacts, the two masses repeat the behavior analyzed above, therefore the mismatch $e$ after the $k$-th impact of $\mathcal{Z}$ can be characterized by $\left(A_{J}\left(\frac{\mu}{v}\right) A_{F}\left(\frac{v}{\mu}\right)\right)^{k} e(0,0)=: A_{J F}\left(\frac{v}{\mu}\right)^{k} e(0,0)$.

The unstable behavior of Example 1 can be avoided by anticipating the fact that future impacts will invert the (normal) speed of the ball, and by enforcing a control strategy in which $\mathcal{X}$ may decide to track either the real reference or the mirrored reference, mirrored through the boundary $F$ as shown in Figure 3, which intuitively reverses the effect of an impact. Mathematically this approach can be enforced by combining a selection policy of the reference to track and (v) of Claim 1, which guarantees that when either $\mathcal{X}$ or $\mathcal{Z}$ impacts the wall $F$ at a point $s \in \mathcal{J}$, it satisfies $m\left(F, s^{+}\right)=m(F, m(F, s))=s$.

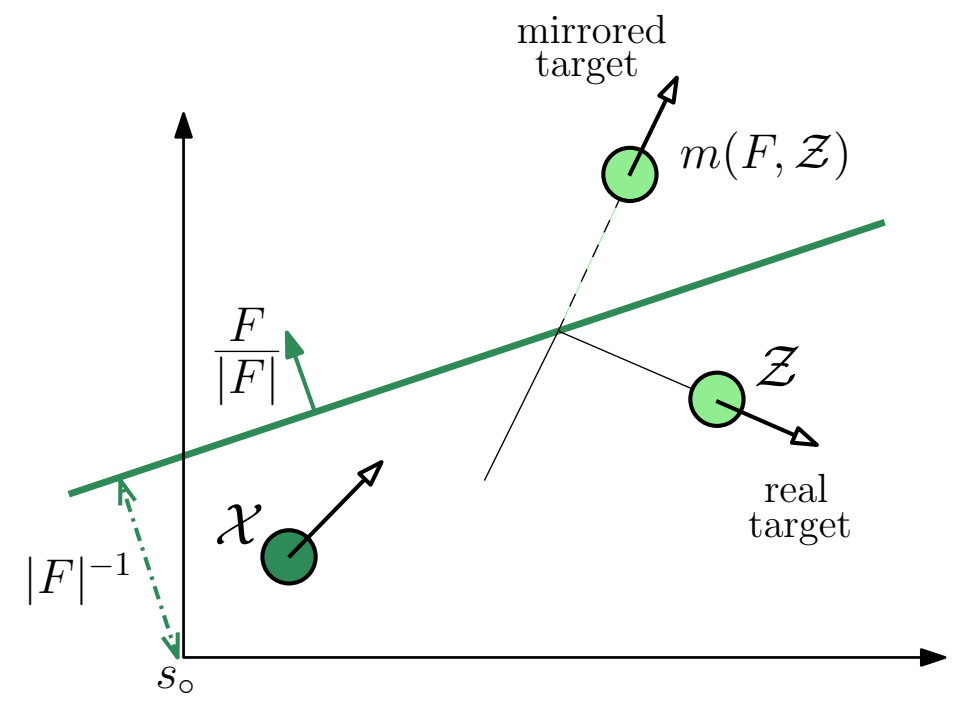

Figure 3. A possible interpretation of the hybrid tracking algorithm.

The control algorithm uses an automaton associated to the index variable $q$ whose dynamics is given by

$$
\dot{q}=0 \quad q \in\{0,1\}
$$




$$
q^{+}=1-q \quad q \in\{0,1\}
$$

which is related to the impacts of $\mathcal{X}$ and $\mathcal{Z}$, since its state is updated only at jumps. Note that $q$ simply toggles between 0 and 1 each time either mass impacts the wall. The control algorithm is parameterized by a vector $K \in \mathbb{R}^{2}$ satisfying the following assumption.

Assumption 2: The gain $K:=\left[\begin{array}{ll}k_{1} & k_{2}\end{array}\right]$ is such that $A_{c l}:=\left[\begin{array}{cc}0 & 1 \\ k_{1} & k_{2}\end{array}\right]$ is Hurwitz.

By introducing the quantities

$$
M(0)=I, \quad c(0)=0, \quad \bar{K}:=K \otimes I_{2},
$$

the control law for a single wall billiard is given by

$$
u=-\phi(x)+M(q F) \alpha+\bar{K}(x-m(q F, z))
$$

where $\alpha \in \alpha(z)$ represents the acceleration of $z_{p}$ at the current time. In particular, $x$ tracks the real target $z$ when $q=0$ since $\bar{K}(x-z)$ is enforced, and it tracks the mirrored target $m(F, z)$ when $q=1$, i.e. $\bar{K}(x-m(F, z))$ is enforced. Since $q$ is toggled at each impact, the transient tracking response depends on the initial value of $q$. For example using $q(0,0)=1$ when $x(0,0)=z(0,0)$ will induce a large initial transient. This transient was avoided in the local solution presented in [7] where $q$ was selected as the minimizer of the function $W$ defined later in (27). Unfortunately such a choice does not induce global results because the minimizer is not well defined globally. Nevertheless, one could select the initial value of $q$ as the minimizer of $W$, thus inducing improved transient responses.

The continuous dynamics of the single-wall tracking closed-loop system is given by (8), (3), (7a), (13a), (15), as summarized below. Let $X=(x, z, \sigma, q), \overline{\mathcal{C}}:=\mathcal{C} \times\{0,1\}$ (see (10)) and $\alpha \in \alpha(z)$ as in (15). For $X \in \overline{\mathcal{C}}$,

$$
\left\{\begin{aligned}
\dot{z} & =\bar{A} z+\bar{B} \alpha \\
\dot{x} & =\bar{A} x+\bar{B}(M(q F) \alpha+\bar{K}(x-m(q F, z))) \\
\dot{q} & =0 \\
\dot{\sigma} & \in[0, \rho] .
\end{aligned}\right.
$$

Based on Section III-A and (13b), the discrete dynamics corresponds to (5), $x^{+}=x$, (7b), (13b) for $(x, z, \sigma, q) \in \overline{\mathcal{D}}_{z}:=\mathcal{D}_{z} \times\{0,1\}$, and to $z^{+}=z$, (9), (7b), (13b) for $(x, z, \sigma, q) \in \overline{\mathcal{D}}_{x}:=\mathcal{D}_{x} \times\{0,1\}$. Defining $\overline{\mathcal{D}}:=\mathcal{D} \times\{0,1\}=\overline{\mathcal{D}}_{z} \cup \overline{\mathcal{D}}_{x}$, (see (11)), it can be summarized as

$$
\begin{aligned}
& \left\{\begin{array}{l}
z^{+}=m(F, z) \\
x^{+}=x \\
q^{+}=1-q \\
\sigma^{+}=\sigma-1
\end{array}, \quad\left\{\begin{array}{l}
z^{+}=z \\
x^{+}=m(F, x) \\
q^{+}=1-q \\
\sigma^{+}=\sigma-1
\end{array},\right.\right. \\
& X \in \overline{\mathcal{D}}_{z}, \quad X \in \overline{\mathcal{D}}_{x},
\end{aligned}
$$

meaning that in $\overline{\mathcal{D}}_{x} \cap \overline{\mathcal{D}}_{z}$ the jump map is the union of the two values above. We can now state the main result on exponential tracking, which is proven in the next section.

Theorem 1: Under Assumptions 1 and 2, there exist $\gamma \geq 1$ and $\lambda>0$ for which each solution $X=$ $(x, z, \sigma, q)$ to the tracking closed-loop system (16) satisfies

$$
\left|x_{p}(t, j)-z_{p}(t, j)\right| \leq \gamma e^{-\lambda(t+j)}|x(0,0)-m(q(0,0) F, z(0,0))|
$$

for all $(t, j) \in \operatorname{dom} X$. 


\section{Closed loop results}

We prove Theorem 1 by showing exponential stability of the compact set

$$
\mathcal{A}:=\left\{(x, z, \sigma, q) \in \mathbb{R}^{4} \times \mathcal{K} \times[0, N] \times\{0,1\} \mid x=m(q F, z)\right\}
$$

according to the following definition.

Definition 1: Consider a hybrid system $\mathcal{H}$ with state $X \in \mathbb{R}^{n}$ and a compact set $\mathcal{A} \subset \mathbb{R}^{n}$. We say that $\mathcal{A}$ is globally exponentially stable (GES) if there exist $\gamma \geq 1$ and $\lambda>0$ such that each solution $X$ to $\mathcal{H}$ satisfies $|X(t, j)|_{\mathcal{A}} \leq \gamma e^{-\lambda(t+j)}|X(0,0)|_{\mathcal{A}}$ for all $(t, j) \in \operatorname{dom} X$.

In fact, we show below in Proposition 1 that $(x, z, \sigma, q) \in \mathcal{A}$ implies $x_{p}=z_{p}$, so that exponentially stabilizing $\mathcal{A}$ implies the result of Theorem 1. Note that Proposition 1 is trivially true if $z_{p}$ is on the boundary of $\mathcal{F}$ where the position is not mirrored. However, we prove it here for all states in $\overline{\mathcal{C}} \cup \overline{\mathcal{D}}$ intersected with $\mathcal{A}$. Then, we introduce some notable identities in Claim 2, which are used in Proposition 2 to show that a suitably defined Lyapunov function $W$ decreases during flows and does not increase at jumps. Combining these preliminary results with [29, Theorem 2], we establish exponential stability of the set $\mathcal{A}$.

Proposition 1: Let $r:=\max _{z \in \mathcal{K}}\{1,|z-m(F, z)|\}$. For the compact set $\mathcal{A}$ defined in (18), for each $X \in \overline{\mathcal{C}} \cup \overline{\mathcal{D}}$,

$$
\left|x_{p}-z_{p}\right| \leq|x-m(q F, z)| \leq r|X|_{\mathcal{A}} \leq r|x-m(q F, z)| .
$$

Moreover, $x=z$ for each $X \in \mathcal{A} \backslash \overline{\mathcal{D}}$.

Proof: Considering $X=(x, z, \sigma, q) \in \overline{\mathcal{C}} \cup \overline{\mathcal{D}}$, which implies $(z, \sigma, q) \in \mathcal{K} \times[0, N] \times\{0,1\}$, from the definition of $\mathcal{A}$ we have

$$
\begin{aligned}
|X|_{\mathcal{A}} & =\min _{\alpha \in \mathcal{K}, \gamma \in[0, N], \beta \in\{0,1\}}\left|\left[\begin{array}{c}
x-m(\beta F, \alpha) \\
z-\alpha \\
\sigma-\gamma \\
q-\beta
\end{array}\right]\right| \\
& \leq\left|\left[\begin{array}{c}
x-m(q F, z) \\
0 \\
0 \\
0
\end{array}\right]\right|=|x-m(q F, z)|,
\end{aligned}
$$

which establishes the last inequality in (19). For the next to last inequality in (19), by using (i) $\left|v_{1}-v_{2}\right|=$ $\left|\bar{M}(\beta F)\left(v_{1}-v_{2}\right)\right|=\left|m\left(\beta F, v_{1}\right)-m\left(\beta F, v_{2}\right)\right|, \forall v_{1}, v_{2} \in \mathbb{R}^{4}, \beta \in\{0,1\}$ which follows from (6d) by (vi) of Claim 1, (ii) $\left|v_{1}-\alpha\right|^{2}+\left|v_{2}-\alpha\right|^{2} \geq\left|v_{1}-v_{2}\right|^{2}, \forall v_{1}, v_{2}, \alpha \in \mathbb{R}^{n}$, and (iii) $r|q-\beta| \geq|m(\beta F, z)-m(q F, z)|$ which holds for all $q, \beta \in\{0,1\}$, we have

$$
\begin{aligned}
|X|_{\mathcal{A}}^{2} & =\min _{\alpha \in \mathcal{K}, \gamma \in[0, N], \beta \in\{0,1\}}\left|\left[\begin{array}{c}
x-m(\beta F, \alpha) \\
z-\alpha \\
\sigma-\gamma \\
q-\beta
\end{array}\right]\right|^{2} \\
& =\min _{\alpha \in \mathcal{K}, \beta \in\{0,1\}}\left|\left[\begin{array}{c}
x-m(\beta F, \alpha) \\
z-\alpha \\
q-\beta \\
x-m(\beta F, \alpha) \\
m(\beta F, z)-m(\beta F, \alpha)
\end{array}\right]\right|^{2} \\
& =\min _{\alpha \in \mathcal{K}, \beta \in\{0,1\}}\left|\left[\begin{array}{c}
q-\beta \\
m(\beta F, z)
\end{array}\right]\right|^{2} \\
& =\min _{\beta \in\{0,1\}} \mid\left[\begin{array}{c}
x-m(\beta F, \\
q-\beta
\end{array}\right] \\
& \geq \min _{\beta \in\{0,1\}} \frac{1}{r^{2}}|x-m(\beta F, z)|^{2}+|q-\beta|^{2} \\
& \geq \min _{\beta \in\{0,1\}} \frac{1}{r^{2}}\left(|x-m(\beta F, z)|^{2}+|m(\beta F, z)-m(q F, z)|^{2}\right) \\
& \geq \frac{1}{r^{2}}|x-m(q F, z)|^{2} .
\end{aligned}
$$

It follows that $|x-m(q F, z)| \leq r|X|_{\mathcal{A}}$ which establishes the next to last inequality in (19).

Finally, for the first inequality in (19), consider the line $\ell$ that connects $z_{p}$ to $M(F) z_{p}+c(F)$ represented by the vector $z_{p}-M(F) z_{p}-c(F)$, and note that this line is perpendicular to the wall $F$. In fact, take 
$F_{\perp} \in \mathbb{R}^{2}$ such that $F_{\perp}^{T} F=0$. Then, using the definitions in (4) and (6),

$$
\begin{aligned}
& F_{\perp}^{T}\left(z_{p}-M(F) z_{p}-c(F)\right)= \\
& =F_{\perp}^{T}\left((I-M(F)) z_{p}-c(F)\right) \\
& =F_{\perp}^{T}\left(R(F)^{T}\left[\begin{array}{ll}
0 & 0 \\
0 & 2
\end{array}\right] R(F) z_{p}-\frac{2 F}{|F|^{2}}\left(1+F^{T} s_{\circ}\right)\right) \\
& =\frac{F_{\perp}^{T}}{|F|^{2}}\left(\left[\begin{array}{ll}
J^{T} F & F
\end{array}\right]\left[\begin{array}{ll}
0 & 0 \\
0 & 2
\end{array}\right]\left[\begin{array}{c}
F^{T} J \\
F^{T}
\end{array}\right] z_{p}-2 F\left(1+F^{T} s_{\circ}\right)\right) \\
& =\frac{F_{\perp}^{T}}{|F|^{2}}\left(2 F F^{T} z_{p}-2 F\left(1+F^{T} s_{\circ}\right)\right) \\
& =\frac{2}{|F|^{2}} F_{\perp}^{T} F\left(F^{T} z_{p}-\left(1+F^{T} s_{\circ}\right)\right)=0 .
\end{aligned}
$$

Moreover, for positions on the wall $F$ given by $\mathcal{S}_{F}:=\left\{s \in \mathbb{R}^{2} \mid F^{T}\left(s-s_{\circ}\right)=1\right\}$, we have

$$
\left|z_{p}\right|_{\mathcal{S}_{F}}=\left|M(F) z_{p}+c(F)\right|_{\mathcal{S}_{F}} .
$$

To see this, note that $\left|z_{p}\right|_{\mathcal{S}_{F}}=\min _{\xi \in \mathcal{S}_{F}}\left|z_{p}-\xi\right|=\left|\frac{F^{T}}{|F|}\left(z_{p}-\xi\right)\right|$ for all $\xi \in \mathcal{S}_{F} \quad$ (where the second identity holds because $\frac{F^{T}}{|F|}\left(z_{p}-\xi\right)$ evaluates the component of the vector $z_{p}-\xi$ that is orthogonal to $\left.\mathcal{S}_{F}\right)$, from which $\left|z_{p}\right|_{\mathcal{S}_{F}}=\left|\frac{F^{T}}{|F|}\left(z_{p}-\xi+s_{\circ}-s_{\circ}\right)\right|=\frac{1}{|F|}\left|F^{T}\left(z_{p}-s_{\circ}\right)-1\right|$. Then, in a similar way, $\left|M(F) z_{p}+c(F)\right|_{\mathcal{S}_{F}}=$ $\frac{1}{|F|}\left|F^{T}\left(M(F) z_{p}+c(F)-s_{\circ}\right)-1\right|=\frac{1}{|F|}\left|-F^{T} z_{p}+2+2 F^{T} s_{0}-F^{T} s_{\circ}-1\right|=\frac{1}{|F|}\left|F^{T}\left(-z_{p}+s_{0}\right)+1\right|=\left|z_{p}\right| \mathcal{S}_{F}$, where in the second identity we used (iii) of Claim 1 and the definition of $c(F)$ in (6). Consider now the set of points that belong to the line $\ell$ from $z_{p}$ to $M(F) z_{p}+c(F)$, denoted by $\mathcal{S}_{\ell}:=\left\{s \in \mathbb{R}^{2} \mid s=\right.$ $\left.\lambda z_{p}+(1-\lambda)\left(M(F) z_{p}+c(F)\right)\right\}$, and define $\eta:=\underset{s \in S_{\ell}}{\operatorname{argmin}}\left|x_{p}-s\right|$, which geometrically is the point of $\mathcal{S}_{\ell}$ on the intersection between the line $\ell$ and the line perpendicular to $\ell$ passing through $x_{p}$ (which is also parallel to the wall $F$ ). We get

$$
\begin{aligned}
\left|x_{p}-z_{p}\right|^{2} & =\left|x_{p}\right|_{\mathcal{S}_{\ell}}^{2}+\left|\eta-z_{p}\right|^{2} \\
\left|x_{p}-M(F) z_{p}-c(F)\right|^{2} & =\left|x_{p}\right|_{\mathcal{S}_{\ell}}^{2}+\left|\eta-M(F) z_{p}-c(F)\right|^{2} .
\end{aligned}
$$

Then, $F^{T}\left(x_{p}-s_{\circ}\right) \leq 1$ guarantees $F^{T}\left(\eta-s_{\circ}\right) \leq 1$, from which $\left|\eta-z_{p}\right|^{2} \leq\left|z_{p}\right|_{\mathcal{S}_{F}}^{2}=\left|M(F) z_{p}+c(F)\right|_{\mathcal{S}_{F}}^{2} \leq$ $\left|\eta-M(F) z_{p}-c(F)\right|^{2}$. It follows that $\left|x_{p}-z_{p}\right| \leq\left|x_{p}-M(q F) z_{p}-c(q F)\right| \leq|x-m(q F, z)|$.

For the last claim of the proposition, take a point $(x, z, \sigma, q) \in \mathcal{A}$ and suppose that $x \neq z$, then $x=m(F, z)$. If $F^{T}\left(z_{p}-s_{\circ}\right)<1$ then $F^{T}\left(x_{p}-s_{\circ}\right)=F^{T}\left(M(F) z_{p}+c(F)-s_{\circ}\right)=-F^{T} z_{p}+2(1+$ $\left.\left.F^{T} s_{\circ}\right)\right)-F^{T} s_{\circ}=-F^{T}\left(z_{p}-s_{\circ}\right)+2>1$, where the second identity follows from (iii) of Claim 1, thus $x$ does not belong to $\mathcal{F}$. In a similar way, if $F^{T}\left(x_{p}-s_{\circ}\right)<1$ then $F^{T}\left(z_{p}-s_{\circ}\right)>1$. The remaining case is $F^{T}\left(z_{p}-s_{\circ}\right)=F^{T}\left(x_{p}-s_{\circ}\right)=1$, and by $x=m(F, z)$ we have that $x_{v}=M(F) x_{v}$, thus $F^{T} x_{v}=-F^{T} z_{v}$, that is, either $x$ or $z$ must belong to $\mathcal{J}$.

Remark 3: The generalization of the set $\mathcal{A}_{\circ}$ defined just before Example 1 to the set $\mathcal{A}=\mathcal{A}_{\circ} \cup \mathcal{A}_{F}$ in (18), where $\mathcal{A}_{\circ}:=\{(x, z, \sigma, q) \mid x=z, q=0\}$ and $\mathcal{A}_{F}:=\{(x, z, \sigma, q) \mid x=m(F, z), q=1\}$, plays a fundamental role in establishing the next result on stability, because the introduction of $\mathcal{A}_{F}$ allows for the invariance of the set $\mathcal{A}$ along the hybrid dynamics, through the relaxation of the constraint $x=z$ at jumps. To appreciate this, consider the example in which $(x, z, \sigma, q)=(\xi, \xi, \sigma, 0) \in \mathcal{A}_{\circ}$ and $\mathcal{Z}$ impacts the wall. Then, $(x, z, \sigma, q)^{+}=(\xi, m(F, \xi), \sigma-1,1) \in \mathcal{A}_{F}$. Moreover, suppose that also $\mathcal{X}$ impacts the wall, then this second jump resets the state to $(m(F, \xi), m(F, \xi), \sigma-2,0) \in \mathcal{A}_{\circ}$.

We prove next two identities about the behavior of the tracking closed-loop system at impacts.

Claim 2: For any given $P=P^{T} \in \mathbb{R}^{2 \times 2}$, define $\bar{P}:=P \otimes I_{2}$. For each $x, z \in \mathbb{R}^{4}$ and $F \in \mathbb{R}^{2}$, (i) $|x-m(F, z)|_{\bar{P}}=|m(F, x)-z|_{\bar{P}}$, (ii) $|m(F, x)-m(F, z)|_{\bar{P}}=|x-z|_{\bar{P}}$.

Proof: Consider the identity $\left(S_{1} \otimes S_{2}\right)\left(S_{3} \otimes S_{4}\right)=S_{1} S_{3} \otimes S_{2} S_{4}$ where $S_{1}, \ldots, S_{4}$ are matrices. For each $S \in \mathbb{R}^{2 \times 2}$ such that $S^{T} S=I$, we have that $\operatorname{diag}\left(S^{T}, S^{T}\right) \bar{P} \operatorname{diag}(S, S)=\left(I_{2} \otimes S^{T}\right)\left(P \otimes I_{2}\right)\left(I_{2} \otimes S\right)=$ $\left[\left(I_{2} P\right) \otimes\left(S^{T} I_{2}\right)\right]\left(I_{2} \otimes S\right)=\left[\left(P I_{2}\right) \otimes\left(I_{2} S^{T}\right)\right]\left(I_{2} \otimes S\right)=\left(P \otimes I_{2}\right)\left(I_{2} \otimes S^{T}\right)\left(I_{2} \otimes S\right)=P \otimes I_{2}=\bar{P}$, thus $\bar{M}(F)^{T} \overline{P M}(F)=\bar{P}$, for each $F \in \mathbb{R}^{2}$. It follows that (i) $|x-m(F, z)|_{\bar{P}}=|x-m(F, z)|_{\bar{M}(F)^{T} \overline{P M}(F)}=$ 
$|\bar{M}(F) x-\bar{M}(F) m(F, z)|_{\bar{P}}=|m(F, x)-z|_{\bar{P}}$, where in the last identity we used (i) and (ii) of Claim 1; and (ii) $|m(F, x)-m(F, z)|_{\bar{P}}=|\bar{M}(F) x-\bar{M}(F) z|_{\bar{P}}=|x-z|_{\bar{M}(F)^{T} \overline{P M}(F)}=|x-z|_{\bar{P}}$.

Remark 4: In the special case commented at the beginning of Section III, when the wall corresponds to the horizontal axis in the plane and $s_{\circ}=\left[\begin{array}{l}0 \\ 1\end{array}\right], F=\left[\begin{array}{c}0 \\ -1\end{array}\right]$, many of the developed derivations simplify

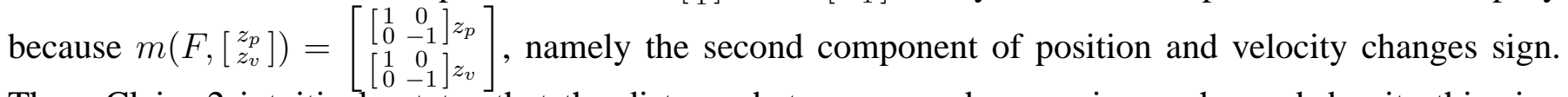
Then, Claim 2 intuitively states that the distance between $x$ and $z$ remains unchanged despite this sign change. Similar intuitive facts also apply to the equalities stated in Claim 1. Finally, the position of any mirrored ball is either at the same position as the original one or in the set where $x_{2}<0$, namely outside the billiard: this is the intuition behind Proposition 1.

Using the following assumption, we build a Lyapunov function $W$ that does not increase at jumps (by Claim 2) while it decreases during flows, by observability (in the linear sense) of $\left(H, A_{c l}\right)$, as specified next.

Assumption 3: The pair of matrices $(P, H)$ is such that

$$
\begin{aligned}
& P=P^{T}>0, \\
& A_{c l}^{T} P+P A_{c l} \leq-H^{T} H, \\
& \text { and }\left(H, A_{c l}\right) \text { is observable, }
\end{aligned}
$$

where $A_{\text {cl }}$ is defined in Assumption 2.

If Assumption 2 holds, there always exists a pair $(P, H)$ that satisfies (25). Define

$$
\bar{P}:=P \otimes I_{2}, \quad \bar{H}:=H \otimes I_{2},
$$

from which we have the following proposition:

Proposition 2: Under Assumption 3 and using (26), consider the function $W: \overline{\mathcal{C}} \cup \overline{\mathcal{D}} \rightarrow \mathbb{R}_{\geq 0}$ given by

$$
W(x, z, \sigma, q)=|x-m(q F, z)|_{P}^{2} .
$$

Then, using $X=(x, z, \sigma, q)$ and $r:=\max _{z \in \mathcal{K}}\{1,|z-m(F, z)|\}$

(i) $\left\{\begin{array}{l}\lambda_{\min }(P)|X|_{\mathcal{A}}^{2} \leq W(x, z, \sigma, q) \\ \lambda_{\max }(P) r^{2}|X|_{\mathcal{A}}^{2} \geq W(x, z, \sigma, q)\end{array} \quad \forall X \in \overline{\mathcal{C}} \cup \overline{\mathcal{D}}\right.$,

(ii) $W(X) \leq-|x-m(q F, z)|_{\bar{H}^{T}}^{2}, \quad \forall X \in \overline{\mathcal{C}}$,

(iii) $W\left(X^{+}\right) \leq W(X) \quad \forall X \in \overline{\mathcal{D}}$.

Proof: From the definition of $W, \lambda_{\min }(P)|x-m(q F, z)|^{2} \leq W(X) \leq \lambda_{\max }(P)|x-m(q F, z)|^{2}$. Then, (i) follows from Proposition 1.

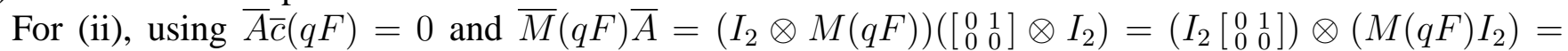
$\left(\left[\begin{array}{ll}0 & 1 \\ 0 & 0\end{array}\right] I_{2}\right) \otimes\left(I_{2} M(q F)\right)=\left(\left[\begin{array}{ll}0 & 1 \\ 0 & 0\end{array}\right] \otimes I_{2}\right)\left(I_{2} \otimes M(q F)\right)=\overline{A M}(q F)$ in the second identity, we get

$$
\begin{aligned}
\dot{W}(X)= & 2(x-m(q F, z))^{T} \bar{P} . \\
& \cdot(\bar{A} x-\bar{M}(q F) \bar{A} z+\overline{B K}(x-m(q F, z))) \\
= & 2(x-m(q F, z))^{T} \bar{P}(\bar{A}+\overline{B K})(x-m(q F, z)) \\
= & 2(x-m(q F, z))^{T}\left(P A_{c l} \otimes I_{2}\right)(x-m(q F, z)) \\
= & -|x-m(q F, z)|_{\bar{H}^{T} \bar{H}}^{2} .
\end{aligned}
$$

(iii) Consider a jump of $\mathcal{Z}$. Using (v) of Claim 1 for $q=0$, and $m(0, m(F, z))=m(F, z)=m(q F, z)$ for $q=1$ (which follows from $M(0)=I$ and $c(0)=0$ ), we get

$$
\begin{aligned}
\left|x^{+}-m\left(q^{+} F, z^{+}\right)\right|_{\bar{P}} & =|x-m((1-q) F, m(F, z))|_{\bar{P}} \\
& =\mid x-m(q F, z))\left.\right|_{\bar{P}} .
\end{aligned}
$$

Consider a jump of $\mathcal{X}$. From (i) of Claim 2, and by using the argument above, we get

$$
\begin{aligned}
\left|x^{+}-m\left(q^{+} F, z^{+}\right)\right|_{\bar{P}} & =|m(F, x)-m((1-q) F, z)|_{\bar{P}} \\
& =\mid x-m(q F, z))\left.\right|_{\bar{P}} .
\end{aligned}
$$


Proposition 2 will be used in the proof of the next theorem to establish global exponential stability of the set $\mathcal{A}$. Subsequently we will prove Theorem 1 and provide some remarks.

Theorem 2: Under Assumption 2, for each $\rho>0$ and $N>0$, the compact set $\mathcal{A}$ is globally exponentially stable for the tracking closed-loop system.

For the proof of Theorem 2, if wanting to establish only global asymptotic stability, we could use Proposition 2, observability of $\left(H, A_{c l}\right)$, the average dwell-time constraint imposed by (7), and the invariance principle [25]. However, since we need to establish global exponential stability, we introduce the following lemma, which is a reformulation of [29, Theorem 2] (see also the proof of [29, Theorem 2]). The lemma will also be used to establish similar results for the observer and the output feedback algorithm of the next sections.

Lemma 1: Consider an observable pair $(H, A)$, with $A \in \mathbb{R}^{n \times n}$, a map $G: \mathbb{R}^{n} \times \mathbb{R}^{m} \rightrightarrows \mathbb{R}^{n}$, and two sets $\mathcal{E}_{c} \subset \mathbb{R}^{n}, \mathcal{E}_{d} \subset \mathbb{R}^{n} \times \mathbb{R}^{m}$. Suppose that there exists a function $V: \mathbb{R}^{n} \rightarrow \mathbb{R}_{\geq 0}$ defined as $V(e):=|e|_{P}^{2}$ for all $e \in \mathbb{R}^{n}$, with $P \in \mathbb{R}^{n \times n}$ symmetric and positive definite, satisfying:

$\left(a_{1}\right)\langle\nabla V(e), A e\rangle \leq-|e|_{H^{T} H}^{2} \quad \forall e \in \mathcal{E}_{c} ;$

$\left(a_{2}\right) \quad V(g) \leq V(e) \quad \forall(e, \xi) \in \mathcal{E}_{d}, \forall g \in G(e, \xi)$.

Then, for each pair $(\rho, N) \in \mathbb{R}_{>0} \times \mathbb{Z}_{>0}$, there exists a function $Y: \mathbb{R}^{n} \times[0, N] \rightarrow \mathbb{R}_{\geq 0}$ and scalars $\lambda_{1}, \lambda_{2}, \lambda_{3}>0, \lambda_{4} \in[0,1)$, such that

(i) $\lambda_{1}|e|^{2} \leq Y(e, \sigma) \leq \lambda_{2}|e|^{2}, \forall \sigma \in[0, N], \forall e \in \mathbb{R}^{n}$

(ii) $\left\langle\nabla_{\sigma} Y(e, \sigma), f\right\rangle+\left\langle\nabla_{e} Y(e, \sigma), A e\right\rangle \leq-\lambda_{3} Y(e, \sigma)$, $\forall \sigma \in[0, N], \forall e \in \mathcal{E}_{c}, \forall f \in[0, \rho]$

(iii) $Y(g, \sigma-1) \leq \lambda_{4} Y(e, \sigma)$, $\forall \sigma \in[1, N], \forall(e, \xi) \in \mathcal{E}_{d}, \forall g \in G(e, \xi)$.

Proof of Theorem 2. Under Assumption 2 we can find a pair of matrices $(P, H)$ that satisfies Assumption 3. Consider the coordinate transformation $(e, \sigma, \xi):=(x-m(q F, z), \sigma,(z, q))$. Then, for each solution $X=(x, z, \sigma, q)$ to the tracking closed loop system, using the new coordinates and Proposition 2, define $V(e)=W(X)$ and note that $\dot{e}=A_{c l} \otimes I_{2}, V(e)=W(X)=|e|_{P}^{2}, \dot{V}(e)=\dot{W}(X)=\left\langle\nabla V(e), A_{c l} \otimes I_{2}\right\rangle \leq$ $-|e|_{\bar{H}^{T} \bar{H}}^{2}$ on flows and $V\left(e^{+}\right)=W\left(X^{+}\right) \leq W(X) \leq V(e)$ on jumps. Thus, for $A$ in Lemma 1 given by $A_{c l} \otimes I_{2}$, and by a suitable definition of $G, \mathcal{E}_{c}$ and $\mathcal{E}_{d}$, each condition of Lemma 1 is satisfied. Therefore, from (i)-(iii) of Lemma 1 and by (i) of Proposition 1, defining $\bar{Y}(X):=Y(e, \sigma)=Y(x-m(q F, z), \sigma)$ and $r:=\max _{z \in \mathcal{K}}\{1,|z-m(F, z)|\}$, we get

- $\lambda_{1}|X|_{\mathcal{A}}^{2} \leq \lambda_{1}|x-m(q F, z)|^{2} \leq \bar{Y}(X) \leq \lambda_{2}|x-m(q F, z)|^{2} \leq \sqrt{2} r \lambda_{2}|X|_{\mathcal{A}}^{2}, \forall X \in \overline{\mathcal{C}} \cup \overline{\mathcal{D}}$;

- $\dot{\bar{Y}}(X) \leq-\lambda_{3} \bar{Y}(X), \forall X \in \overline{\mathcal{C}}$;

- $\bar{Y}\left(X^{+}\right) \leq \lambda_{4} \bar{Y}(X), \forall X \in \overline{\mathcal{D}}$,

which, according to [29, Theorem 1], establish global exponential stability of the set $\mathcal{A}$ in (18).

Proof of Theorem 1. The proof follows from Proposition 1 and Theorem 2.

Remark 5: For given $(\rho, N)$, the average dwell-time automaton (7) may terminate prematurely solutions that start from points where the initial value of $x_{0}$ is very large since such solutions may produce a large number of impacts before settling into tracking. Nevertheless, because of the independence of the stability result from the parameter selection, premature termination can be addressed by selecting $\rho$ and $N$ sufficiently large, based on the size of $x_{0}$.

Remark 6: Given $A_{c l}$ Hurwitz, according to Assumption 3, the results in Proposition 2 are obtained for pairs $(P, H)$ such that $A_{c l}^{T} P+P A_{c l} \leq-H^{T} H$ with $\left(H, A_{c l}\right)$ observable. The generality of allowing $H^{T} H \geq 0$ instead of requiring $H^{T} H>0$ will be exploited in next section to analyze some specific multiple-wall billiards (parallel walls) for which the nonincreasing feature of the function $W$ at jumps cannot be guaranteed by any $P$ which satisfies $A_{c l}^{T} P+P A_{c l}<0$.

Remark 7: The hybrid dynamics of the two translating masses, the control algorithm presented in Sections II and III, and the analysis performed above can be generalized to spaces of higher dimension. 
For example, the whole approach can be lifted to a three-dimensional space, with impacts occurring on walls (planes) denoted by vectors $F \in \mathbb{R}^{3}$. Indeed, the mirroring function $m(F, z)$ would preserve the structure given in (4), since it inverts only the component of the velocity subvector parallel to $F$ (orthogonal to the plane).

Remark 8: The effect of small delays on impact detection can be modeled by replacing $q$ in (15) with a new logic variable $\tilde{q}$, whose value is updated to the value of $q$ after a delay bounded by $\Delta>0$, which produces a bounded perturbation of duration shorter than or equal to $\Delta$ on the input $u$ after each impact ( $q$ is constant between impacts). The analysis of the perturbed system can then be developed within the robustness framework on hybrid systems [11], [26], [24], to show that the perturbation on $u$ produces perturbed trajectories $x_{\Delta}$ whose distance from the desired trajectories $x$, in a graphical sense [12], shrinks to zero as $\Delta$ goes to zero, which leads to practical stability results, i.e. convergence of solutions to the set $\mathcal{A}+\gamma(\Delta) \mathbb{B}$, where $\gamma$ is a continuous function, strictly increasing and such that $\gamma(0)=0$. These considerations also apply to the observer and output feedback controller designs of the next sections. $\lrcorner$

\section{OBSERVER CONSTRUCTION: SINGLE WALL}

\section{A. Observer structure and single wall algorithm}

We consider the problem of designing an observer $\mathcal{X}$ to estimate the state of $\mathcal{Z}$ from the output $y=C z$, $C:=[I \mid 0] \in \mathbb{R}^{2 \times 4}$, i.e. when the speed $z_{v}$ is not available for measurement.

We replace the continuous dynamics (3) of the reference system $\mathcal{Z}$ by

$$
\mathcal{Z} \quad: \quad \dot{z} \in \bar{A} z+\bar{B} \alpha(C z), \quad z \in \mathcal{K}
$$

which differs from (3) only for the restriction of the set-valued map $\alpha: \mathbb{R}^{2} \rightrightarrows \mathbb{R}^{2}$, which is now an outer semicontinuous and locally bounded set-valued map having nonempty convex values for each $z_{p}=C z \in \Pi_{p}(\mathcal{F}):=\left\{z_{p} \mid z \in \mathcal{F}\right\}$. As before, we allow for set-valued accelerations for the reference variable $z_{p}$ but the selected acceleration at each time is assumed to be known by the observer algorithm.

The observer continuous dynamics is given by

$$
\mathcal{X}: \dot{x}=\bar{A} x+u, \quad x \in \mathcal{F}
$$

where here the action of $u=\left[u_{p}^{T} \mid u_{v}^{T}\right]^{T} \in \mathbb{R}^{4}$ affects both $\dot{x}_{p}$ and $\dot{x}_{v}$. The jump dynamics of the observer resembles the impact dynamics of the tracking case, and is given by

$$
\mathcal{X}: x^{+} \in \bigcup_{i \in \mathcal{M}(x)} m\left(F_{i}, x\right), \quad\left(x_{p}, x_{v}+u_{p}\right) \in \mathcal{J}
$$

which differs from (9) due to the definition of the jump condition, which now explicitly depends on the input subvector $u_{p}$. In fact, the dynamics $\dot{x}_{p}=x_{v}$ of the tracking approach is now replaced by $\dot{x}_{p}=x_{v}+u_{p}$, from which the impact condition $\left\langle F_{i}, \dot{x}_{p}\right\rangle=\left\langle F_{i}, x_{v}\right\rangle \geq 0$ is replaced by $\left\langle F_{i}, \dot{x}_{p}\right\rangle=\left\langle F_{i}, x_{v}+u_{p}\right\rangle \geq 0$.

Remark 9: Although the jump dynamics of the observer is not necessarily connected to the impacts physics of the billiard (no "physical" walls are impacted by the observer), with the new definition of the jump set, we preserve the analogy with the tracking case, enforcing a reset behavior of the observer that resembles the impact behavior of a translating mass whose velocity is given by $\dot{x}_{p}=s_{1}+s_{2}$, with $s_{1}=x_{v}$ and $s_{2}=u_{p}$. Note that when $u_{p}=0$, the jump dynamics of $\mathcal{X}$ (both the jump set and the jump map) coincides with the jump dynamics of $\mathcal{Z}$.

Following the approach of Section III-B, the observer algorithm is parameterized by a vector $L \in \mathbb{R}^{2}$ satisfying the following assumption.

Assumption 4: The gain $L=\left[\begin{array}{ll}\ell_{1} & \ell_{2}\end{array}\right]^{T}$ is such that $A_{c l}:=\left[\begin{array}{ll}\ell_{1} & 1 \\ \ell_{2} & 0\end{array}\right]$ is Hurwitz.

Thus, using (4) and (6) and $\bar{L}:=L \otimes I_{2}$, the input $u$ for a single wall billiard is given by

$$
\begin{aligned}
u & =\bar{M}(q F) \bar{B} \alpha+\bar{L}(C x-M(q F) C z-c(q F)) \\
& =\bar{M}(q F) \bar{B} \alpha+\bar{L} C(x-m(q F, z)),
\end{aligned}
$$


where $\alpha \in \alpha(C z)$ is the acceleration of $z_{p}$, and the observer closed-loop system has flow dynamics given by (31), (30), (7a), (13a), and (33), which is enabled for $(x, z, \sigma, q) \in \overline{\mathcal{C}}$, where

$$
\overline{\mathcal{C}}:=\mathcal{F} \times \mathcal{K} \times[0, N] \times\{0,1\},
$$

while the jump dynamics is given by (32), $z^{+}=z$, (7b), (13b) when $\left(\left(x_{p}, x_{v}+u_{p}\right), z, \sigma, q\right) \in \overline{\mathcal{D}}_{x}$, and by $x^{+}=x,(5),(7 \mathrm{~b}),(13 \mathrm{~b})$ when $(x, z, \sigma, q) \in \overline{\mathcal{D}}_{z}$, where

$$
\begin{aligned}
\overline{\mathcal{D}}_{x} & :=\mathcal{J} \times \mathcal{K} \times[1, N] \times\{0,1\}, \\
\overline{\mathcal{D}}_{z} & :=\mathcal{F} \times(\mathcal{J} \cap \mathcal{K}) \times[1, N] \times\{0,1\}, \\
\overline{\mathcal{D}} & =\overline{\mathcal{D}}_{x} \cup \overline{\mathcal{D}}_{z} .
\end{aligned}
$$

\section{B. Closed-loop results}

Following the approach of Section III-C, we state below the main result of the current section, on stability of the set $\mathcal{A}$ defined in (18).

Theorem 3: Under Assumption 4, for each $\rho>0$ and $N>0$, the compact set $\mathcal{A}$ is globally exponentially stable for the observer closed-loop system.

Proof: Under Assumption 4, consider two matrices $(P, H)$ satisfying (25) with $A_{c l}=\left[\begin{array}{ll}\ell_{1} & 1 \\ \ell_{2} & 0\end{array}\right]$ and define $W: \overline{\mathcal{C}} \cup \overline{\mathcal{D}} \rightarrow \mathbb{R}_{\geq 0}$ as $W(x, z, \sigma, q)=|x-m(q F, z)|_{\bar{P}}^{2}$. Then, the function $W$ and the jump dynamics of the observer closed-loop system satisfy statements (i) and (iii) of Proposition 2, which can be established by following exactly the argument proposed at points (i) and (iii) of the proof of Proposition 2. Moreover, following (ii) of the proof of Proposition 2, using the relations $\bar{A} \bar{c}(q F)=0$ and $\bar{M}(q F) \bar{A}=\overline{A M}(q F)$ in the second identity below, we get

$$
\begin{aligned}
\dot{W}(X)= & 2(x-m(q F, z))^{T} \bar{P} . \\
& \cdot(\bar{A} x+\bar{L}(C x-C m(q F, z))-\bar{M}(q F) \bar{A} z) \\
= & 2(x-m(q F, z))^{T} \bar{P}(\bar{A}+\bar{L} C)(x-m(q F, z)) \\
\leq & 2(x-m(q F, z))^{T}\left(P A_{c l} \otimes I_{2}\right)(x-m(q F, z)) \\
\leq & -|x-m(q F, z)|_{\bar{H}^{T}}^{2} .
\end{aligned}
$$

Then, global exponential stability follows from Lemma 1 and [29, Theorem 1] using the coordinate transformation $(e, \sigma, \xi):=(x-m(q F, z), \sigma,(z, q))$, as in the proof of Theorem 2.

Remark 10: The second identity in (36) follows from $\bar{M}(q F) \bar{A} z=\overline{A M}(q F) z=\bar{A}(\bar{M}(q F) z+\bar{c}(F)$ ), since $\bar{A} \bar{c}(q F)=0$ (with $\bar{A}$ given after (8)). These identities also hold when $\bar{A}$ is of the form $\left[\begin{array}{c|c}0 & I \\ \hline 0 & A_{v}\end{array}\right]$, $A_{v} \in \mathbb{R}^{2 \times 2}$, while they do not hold when $\bar{A}$ is of the form $\left[\begin{array}{c|c}0 & I \\ \hline A_{p} & A_{v}\end{array}\right], A_{p}, A_{v} \in \mathbb{R}^{2 \times 2}, A_{p} \neq 0$. However, this type of dynamics can still be described by our model because $A_{p} z_{p}$ can be accounted for within the function $\alpha(C z)$.

The combination of the jump set in (32) and of $u$ in (33) guarantees that if $\left(x_{p}, x_{v}+u_{p}\right) \in \mathcal{J}$ with $\left\langle F, x_{v}+u_{p}\right\rangle>0$, then $\left(x_{p}, x_{v}+u_{p}\right)^{+} \notin \mathcal{J}$, as established in the next proposition. This guarantees that no Zeno solutions are induced by the observer algorithm. In fact, the dwell-time automaton $\sigma$ has been introduced in Section II to rule out trajectories that impact a wall with null normal component, i.e. $\left\langle F, \dot{x}_{p}\right\rangle=\left\langle F, x_{v}+u_{p}\right\rangle=0$, that is usually associated with a translating mass sliding along the wall, and for which the connected Zeno phenomena can be essentially considered as a mathematical side-effect of the particular model adopted. Proposition 3 guarantees that also for the observer closed-loop system the dwell-time automaton only operates on those trajectories, since the jump dynamics (32) does not introduce new Zeno phenomena.

Proposition 3: For the observer closed-loop system, if $\left\langle F, x_{p}-s_{\circ}\right\rangle=1$ and $\left\langle F, x_{v}+u_{p}\right\rangle>0$ then $\left\langle F,\left(x_{v}+u_{p}\right)^{+}\right\rangle<0$.

Proof: Suppose $F^{T}\left(x_{p}-s_{\circ}\right)=1$ and $F^{T}\left(x_{v}+u_{p}\right)=F^{T}\left(x_{v}+\ell_{1}\left[x_{p}-M(q F) z_{p}-c(q F)\right]\right)>0$. In this case, $x_{p}^{+}=M(F) x_{p}+c(F)=x_{p}$ (by (iv) of Claim 1) and $z_{p}^{+}=z_{p}$ (no jump). For the case $q=0$, 
$q^{+}=1$, using $M(q F)=M(0)=I$ and $c(q F)=c(0)=0$ in the next to last identity, and (iii) of Claim 1 in the last identity, we have

$$
\begin{aligned}
& F^{T}\left(x_{v}+u_{p}\right)^{+}= \\
& =F^{T}\left(x_{v}^{+}+\ell_{1}\left[x_{p}^{+}-M\left(q^{+} F\right) z_{p}^{+}-c\left(q^{+} F\right)\right]\right) \\
& =F^{T}\left(M(F) x_{v}+\ell_{1}\left[M(F) x_{p}+c(F)-M(F) z_{p}-c(F)\right]\right) \\
& =F^{T} M(F)\left(x_{v}+\ell_{1}\left[x_{p}-z_{p}\right]\right) \\
& =F^{T} M(F)\left(x_{v}+\ell_{1}\left[x_{p}-M(q F) z_{p}-c(q F)\right]\right) \\
& =-F^{T}\left(x_{v}+u_{p}\right) .
\end{aligned}
$$

For the case $q=1, q^{+}=0$, using in the third identity (i) of Claim 1 and $F^{T} c(F)=-F^{T} M(F) c(F)$ (by (iii) of Claim 1), we have

$$
\begin{aligned}
& F^{T}\left(x_{v}+u_{p}\right)^{+}= \\
& =F^{T}\left(x_{v}^{+}+\ell_{1}\left[x_{p}^{+}-M\left(q^{+} F\right) z_{p}^{+}-c\left(q^{+} F\right)\right]\right) \\
& =F^{T}\left(M(F) x_{v}+\ell_{1}\left[M(F) x_{p}+c(F)-z_{p}\right]\right) \\
& =F^{T} M(F)\left(x_{v}+\ell_{1}\left[x_{p}-c(F)-M(F) z_{p}\right]\right) \\
& =F^{T} M(F)\left(x_{v}+\ell_{1}\left[x_{p}-M(q F) z_{p}-c(q F)\right]\right) \\
& =-F^{T}\left(x_{v}+u_{p}\right) .
\end{aligned}
$$

Summarizing, Theorem 3 establishes global exponential stability of $\mathcal{A}$ which, by Proposition 1 , corresponds to the set where $x=z$ (zero observation error), except for the hybrid times when jumps occur. ${ }^{4}$ Moreover, Proposition 3 guarantees that when the observer mass $\mathcal{X}$ impacts a wall after the arising jump, the mass is reflected back toward the interior of the billiard $\mathcal{F}$.

\section{SPeCIAL BILliaRdS With MULTIPLE WALLS}

\section{A. Tracking and observer closed-loop systems for billiards with multiple-walls}

Henceforth, we generalize the global results on stability of the previous sections to multiple-wall billiards having specific polyhedral shapes. This section is connected with the local tracking technique presented in [7], [8], which is developed for general billiards, i.e. billiards with a locally Lipschitz boundary (like polyhedra), and with the global tracking technique presented in [8], which proposes a Lyapunov-based selection policy between mirrored and real targets.

The first step toward the generalization of the results of the previous sections is the redefinition of the input $u$ in (15) and (33). For the billiard $\mathcal{F}$ in (1) with walls $F_{i}$ with $i \in \mathcal{I}:=\{1, \ldots, r\} \subset \mathbb{N}$, define $F_{0}:=0$ and $\overline{\mathcal{I}}:=\{0\} \cup \mathcal{I}$, and consider an automaton generalizing that in (13) and given by

$$
\begin{aligned}
\dot{q} & =0, & q \in \mathcal{Q} \\
q^{+} & =\delta(q, i), & q \in \mathcal{Q},
\end{aligned}
$$

where $\delta: \mathcal{Q} \times \overline{\mathcal{I}} \rightarrow \mathcal{Q}$ is a function whose definition depends on the particular shape of the billiard (it will be characterized in next sections), where the state variable $q$ belongs to a given set $\mathcal{Q} \subset \mathbb{N}, \overline{\mathcal{I}} \subset \mathcal{Q}$, and the input $i$ is given by the wall impacted by either $\mathcal{Z}$ or $\mathcal{X}$. Consider also the following quantities: for $i \in \overline{\mathcal{I}}$ and $s \in \mathbb{R}^{4}$, generalizing those in (6):

$$
\begin{gathered}
M_{i}:=M\left(F_{i}\right), \bar{M}_{i}:=\bar{M}\left(F_{i}\right), \\
\bar{c}_{i}:=\bar{c}\left(F_{i}\right), \text { and } m_{i}(s):=m\left(F_{i}, s\right) .
\end{gathered}
$$

\footnotetext{
${ }^{4}$ Indeed, at those times, $\mathcal{A}$ allows for an instantaneous mismatch of the speeds $\left(x_{v}=M(F) z_{v} \neq z_{v}\right)$ arising from a pair of consecutive jumps occurring at the same ordinary time $t$. For example, if $\mathcal{Z}$ jumps first, then $\left(x_{v}(t, j), z_{v}(t, j)\right)=\left(\bar{z}_{v}, \bar{z}_{v}\right) \rightarrow\left(x_{v}(t, j+1), z_{v}(t, j+1)\right)=$ $\left(\bar{z}_{v}, M(F) \bar{z}_{v}\right) \rightarrow\left(x_{v}(t, j+2), z_{v}(t, j+2)\right)=\left(M(F) \bar{z}_{v}, M(F) \bar{z}_{v}\right)$
} 
Moreover, for $i \in \mathcal{Q} \backslash \overline{\mathcal{I}}$ and $s \in \mathbb{R}^{4}$, consider new matrices $M_{i} \in \mathbb{R}^{2 \times 2}$ and $c_{i} \in \mathbb{R}^{2}$ (to be chosen), and define

$$
\bar{M}_{i}:=\operatorname{diag}\left(M_{i}, M_{i}\right), \bar{c}_{i}:=\left[c_{i}^{T} \mid 0_{1 \times 2}\right]^{T}, m_{i}(s):=\bar{M}_{i} s+\bar{c}_{i},
$$

where, intuitively, for each $i \in \mathcal{I}, m_{i}(s)$ is the "mirroring" of $s$ through the $i$ th wall, while for $i \in \mathcal{Q} \backslash \overline{\mathcal{I}}$, the map $s \mapsto m_{i}(s)$ will be used for tracking or observing extra mirrored targets constructed as the "mirroring of the mirroring" of the real target, that is, based on the composition of the mirroring transformation in (4), applied to different walls. Then, the control input $u$ is given by

$$
\begin{aligned}
& u=u_{c}:=-\phi(x)+M_{q} \alpha+\bar{K}\left(x-m_{q}(z)\right) \\
& u=u_{o}:=\bar{M}_{q} \bar{B} \alpha+\bar{L}\left(C x-C m_{q}(z)\right),
\end{aligned}
$$

respectively, for the tracking and state-estimation cases, where, as before, either $\alpha \in \alpha(z)$ in (40a) or $\alpha \in \alpha(C z)$ in (40b) represents the acceleration of $z_{p}$ at the current time, $\bar{K}:=K \otimes I_{2}, \bar{L}:=L \otimes I_{2}$, and $K$ and $L$ satisfy Assumptions 2 and 4, respectively. Thus, for multiple-wall billiards,

- the tracking closed-loop system has the flow dynamics given by (8), (3), (7a), (37a), (40a), which is enabled for $(x, z, \sigma, q) \in \overline{\mathcal{C}}$, where

$$
\overline{\mathcal{C}}:=\mathcal{F} \times \mathcal{K} \times[0, N] \times \mathcal{Q},
$$

while its jump dynamics is given by (9), $z^{+}=z$, (7b), (37b), $i \in \mathcal{M}(x)$, when $(x, z, \sigma, q) \in \overline{\mathcal{D}}_{x}$, and by $x^{+}=x,(5),(7 \mathrm{~b}),(37 \mathrm{~b}), i \in \mathcal{M}(z)$, when $(x, z, \sigma, q) \in \overline{\mathcal{D}}_{z}$, where

$$
\begin{aligned}
\overline{\mathcal{D}}_{x} & :=\mathcal{J} \times \mathcal{K} \times[1, N] \times \mathcal{Q}, \\
\overline{\mathcal{D}}_{z} & :=\mathcal{F} \times(\mathcal{J} \cap \mathcal{K}) \times[1, N] \times \mathcal{Q}, \\
\overline{\mathcal{D}} & :=\overline{\mathcal{D}}_{x} \cup \overline{\mathcal{D}}_{z} ;
\end{aligned}
$$

- the observer closed-loop system has the flow dynamics given by (31), (30), (7a), (37a), (40b), which is enabled for $(x, z, \sigma, q) \in \overline{\mathcal{C}}$, with $\overline{\mathcal{C}}$ in (41), while its jump dynamics is given by (32), $z^{+}=z,(7 \mathrm{~b})$, (37b), $i \in \mathcal{M}(x)$, when $\left(\left(x_{p}, x_{v}+u_{p}\right), z, \sigma, q\right) \in \overline{\mathcal{D}}_{x}$, and by $x^{+}=x$, (5), (7b), (37b), $i \in \mathcal{M}(z)$, when $(x, z, \sigma, q) \in \overline{\mathcal{D}}_{z}$, with $\overline{\mathcal{D}}_{x}$ and $\overline{\mathcal{D}}_{z}$ in (42).

\section{B. Closed-loop results}

Following the approach of Section III, we consider the compact set $\mathcal{A}$ given by

$$
\mathcal{A}:=\left\{(x, z, \sigma, q) \in \mathbb{R}^{4} \times \mathcal{K} \times[0, N] \times \mathcal{Q} \mid x=m_{q}(z)\right\},
$$

and we make the following assumption, needed to show several results below, which restrict the analysis proposed below to specific classes of billiards.

Assumption 5: For all $X=(x, z, \sigma, q) \in \mathcal{A}$, if $X \in \overline{\mathcal{C}} \cup \overline{\mathcal{D}}$ then $q \in \overline{\mathcal{I}}$.

Remark 11: Assumption 5 holds for many interesting cases, including two parallel walls, two perpendicular walls, and rectangles as presented in Section V-C. Assumption 5 is typically established by noticing that $z_{p}$ is never at a corner point of the billiard when $z \in \mathcal{K}$ (by Assumption 1), and then showing that if $z_{p}$ is not at a corner, then $x \neq m_{i}(z)$ for $i \in \mathcal{Q} \backslash \overline{\mathcal{I}}$.

Now, paralleling Proposition 1 , we show that $(x, z, \sigma, q) \in \mathcal{A}$ implies $x_{p}=z_{p}$, and $(x, z, \sigma, q) \in \mathcal{A} \backslash \overline{\mathcal{D}}$ implies $x=z$, and finally we prove that $\mathcal{A}$ is globally exponentially stable for the tracking closed-loop system and the observer closed-loop system defined above.

Proposition 4: Let $r:=\max _{z \in \mathcal{K}, \beta, q \in \mathcal{Q}}\left\{1,\left|m_{\beta}(z)-m_{q}(z)\right|\right\}$. Under Assumption 5, for the compact set $\mathcal{A}$ defined in (43),

$$
\left|x-m_{q}(z)\right| \leq r|X|_{\mathcal{A}} \leq r\left|x-m_{q}(z)\right|,
$$

for each $X=(x, z, \sigma, q) \in \overline{\mathcal{C}} \cup \overline{\mathcal{D}}$. Moreover, there exists $\varepsilon>0$ such that for each $X=(x, z, \sigma, q) \in \overline{\mathcal{C}} \cup \overline{\mathcal{D}}$ if $q \in \overline{\mathcal{I}}$ or $|X|_{\mathcal{A}}<\varepsilon$ then

$$
\left|x_{p}-z_{p}\right| \leq\left|x-m_{q}(z)\right|
$$


Finally, for each $X \in \mathcal{A} \backslash \overline{\mathcal{D}}, x=z$.

Proof: To establish the inequalities $\left|x-m_{q}(z)\right| \leq r|X|_{\mathcal{A}} \leq r\left|x-m_{q}(z)\right|$, consider (20)-(21). Then, the result follows by replacing $\beta \in\{0,1\}$ with $\beta \in \mathcal{Q}, m(\beta F, \alpha)$ with $m_{\beta}(\alpha)$, and $m(q F, \alpha)$ with $m_{q}(\alpha)$ in those equations. For inequality (45), consider $q \in \overline{\mathcal{I}}$. We can repeat the proof of the first inequality of Proposition 1, getting $\left|x_{p}-z_{p}\right| \leq\left|x-m_{q}(z)\right|$ for all $q \in \overline{\mathcal{I}}$. Consider now the other case. By Assumption 5, for each $X=(x, z, \sigma, q) \in \mathcal{A} \cap(\overline{\mathcal{C}} \cup \overline{\mathcal{D}})$, $q$ belongs to $\overline{\mathcal{I}}$, therefore (i) for each $s \in \mathcal{Q} \backslash \overline{\mathcal{I}}, x \neq m_{s}(z)$, i.e. $\left|x-m_{s}(z)\right|>0$, and (ii) $\left|x_{p}-z_{p}\right|=0$ since $\left|x_{p}-z_{p}\right| \leq\left|x-m_{q}(z)\right| \leq r|X|_{\mathcal{A}}=0$. Therefore, for each $X \in \mathcal{A} \cap(\overline{\mathcal{C}} \cup \overline{\mathcal{D}}),\left|x_{p}-z_{p}\right|<\left|x-m_{s}(z)\right|$. Then, using the continuity of $m_{s}(z)$ for $s \in \mathcal{Q} \backslash \overline{\mathcal{I}}$, there exists $\varepsilon>0$ (sufficiently small) such that for each $s \in \mathcal{Q} \backslash \overline{\mathcal{I}}$ and each $X \in(\mathcal{A}+\varepsilon \mathbb{B}) \cap(\overline{\mathcal{C}} \cup \overline{\mathcal{D}})$, it holds that $\left|x_{p}-z_{p}\right| \leq\left|x-m_{s}(z)\right|$. In fact, suppose that this claim is false so that for each positive integer $i$ there exist $X_{i} \in\left(\mathcal{A}+\frac{1}{1+i} \mathbb{B}\right) \cap(\overline{\mathcal{C}} \cup \overline{\mathcal{D}})$, such that $\left|x_{p_{i}}-z_{p_{i}}\right|>\left|x_{i}-m_{s_{i}}\left(z_{i}\right)\right|$. Then, there exists a subsequence which converges to a point $X^{*} \in \mathcal{A} \cap(\overline{\mathcal{C}} \cup \overline{\mathcal{D}})$ such that $\left|x_{p}^{*}-z_{p}^{*}\right| \geq\left|x^{*}-m_{s^{*}}\left(z^{*}\right)\right|$ which contradicts the fact established above that $\left|x_{p}-z_{p}\right|<\left|x-m_{s}(z)\right|$ for each $X \in \mathcal{A} \cap(\overline{\mathcal{C}} \cup \overline{\mathcal{D}})$. Finally, using Assumption 5, the claim $x=z$ for each $X \in \mathcal{A} \backslash \overline{\mathcal{D}}$ can be proved using the same argument of Proposition 1.

We can now state the main results of this section on global exponential stability of the set $\mathcal{A}$. The next two theorems are based on a specific condition at jumps (46), which can be satisfied for several cases including two parallel walls, two perpendicular walls, and rectangles, as shown in Section V-C.

Theorem 4: Under Assumption 2 and 5 consider a pair $(P, H)$ that satisfies (25) for $A_{c l}=\left[\begin{array}{cc}0 & 1 \\ k_{1} & k_{2}\end{array}\right]$, and define $\bar{P}:=P \otimes I_{2}$. If for each $(x, z, \sigma, q) \in \overline{\mathcal{D}}$,

$$
\left|x^{+}-m_{q^{+}}\left(z^{+}\right)\right|_{\bar{P}} \leq\left|x-m_{q}(z)\right|_{\bar{P}}
$$

then for each $\rho>0$ and $N>0, \mathcal{A}$ is GES for the tracking closed-loop system.

Theorem 5: Under Assumptions 4 and 5 consider a pair $(P, H)$ that satisfies (25) for $A_{c l}=\left[\begin{array}{ll}\ell_{1} & 1 \\ \ell_{2} & 0\end{array}\right]$, and define $\bar{P}:=P \otimes I_{2}$. If (46) is satisfied for each $(x, z, \sigma, q) \in \overline{\mathcal{D}}$, then for each $\rho>0$ and $N>0, \mathcal{A}$ is GES for the observer closed-loop system.

Proof of Theorems 4 and 5. We develop the analysis of the tracking system using the function $W=$ $\left|x-m_{q}(z)\right|_{\bar{P}}^{2}$. Under the assumptions of Theorem 4, following the approach of the proof of Proposition 2, $W$ satisfies (i)-(iii) in Proposition 2, with $m(q F, z)$ in Proposition 2 replaced by $m_{q}(z)$, and with $r$ defined in Proposition 4. Then, to achieve global exponential stability of the set $\mathcal{A}$ we invoke Lemma 1 and [29, Theorem 1], using the coordinate transformation $(e, \sigma, \xi):=\left(x-m_{q}(z), \sigma,(z, q)\right)$ as shown in the proof of Theorem 2. The proof of Theorem 5 follows similar steps.

\section{Sufficient conditions for Theorems 4 and 5}

The cases of a single wall presented in Sections III and IV satisfy the assumptions of Theorems 4 and 5 with $\mathcal{Q}=\overline{\mathcal{I}}=\{0,1\}$ and $\delta(q, i)$ defined by $\delta(q, i):=1-q$, for $(q, i) \in \overline{\mathcal{I}}$ (indeed, this definition of $\delta$ coincides with the update rule for $q$ used in (13)).

Proposition 5 (Two parallel walls): Consider $F_{1}, F_{2} \in \mathbb{R}^{2}$ such that $\frac{F_{1}}{\left|F_{1}\right|}=-\frac{F_{2}}{\left|F_{2}\right|}, \overline{\mathcal{I}}:=\{0,1,2\}$, and define $\mathcal{Q}:=\overline{\mathcal{I}}$ and assume that $P>0$ is a diagonal matrix. Then, Assumption 5 is trivially satisfied and (46) is satisfied for a function $\delta$ given by (see also Figure 4)

$$
\begin{aligned}
& \delta(0,1):=1, \quad \delta(0,2):=2, \quad \delta(q, i):=0 \text { for } q, i \in\{1,2\} . \\
& \begin{array}{c|ll}
\mathbf{q} \backslash \mathbf{i} & 1 & 2 \\
\hline 0 & 1 & 2 \\
1 & 0 & 0 \\
2 & 0 & 0
\end{array} \quad \text { (1) } \underset{1}{\stackrel{1,2}{\rightleftarrows}} \stackrel{2}{\underset{1,2}{\stackrel{2}{\longrightarrow}}} \text { (2) }
\end{aligned}
$$

Figure 4. Definition of $\delta$ for two parallel walls. The node labels represent $q$ while the arcs labels represent $i$. The direction of the arcs points to the value $\delta(q, i)$, namely, the update law $q^{+}$in (37b). 
Proof: We have to analyze only the case $q \in\{1,2\}$ when either $\mathcal{X}$ or $\mathcal{Z}$ impacts the $i$ th wall with $i \neq q$, since the remaining configurations have been analyzed in the one-wall case of the previous sections. Consider an impact of $\mathcal{Z}$ on the $i$ th wall, $i \neq q$, and $i, q \in\{1,2\}$. We have,

$$
\begin{aligned}
\left|x^{+}-m_{q^{+}}\left(z^{+}\right)\right|_{\bar{P}} & =\left|x-m_{\delta(q, i)}\left(m_{i}(z)\right)\right|_{\bar{P}} \\
& =\left|x-m_{i}(z)\right|_{\bar{P}} \\
& \leq\left|x-m_{q}(z)\right|_{\bar{P}}
\end{aligned}
$$

where the last inequality can be established by using the fact that $F_{1}$ and $F_{2}$ are parallel. Indeed, given the diagonal structure of $\bar{P}$, we have that $\left|x-m_{i}(z)\right|_{\bar{P}}-\left|x-m_{q}(z)\right|_{\bar{P}}$ depends on the differences $\left|x_{p}-M_{i} z_{p}-c_{i}\right|-\left|x_{p}-M_{q} z_{p}-c_{q}\right|$ and $\left|x_{v}-M_{i} z_{v}\right|-\left|x_{v}-M_{q} z_{v}\right|$. For the second difference, since $F_{1}$ and $F_{2}$ are parallel, $M_{i}=M_{q}$, from which $M_{i} z_{v}=M_{q} z_{v}$, thus $\left|x_{v}-M_{i} z_{v}\right|-\left|x_{v}-M_{q} z_{v}\right|=0$. For the first difference, note that

1) $\left|x_{p}-M_{i} z_{p}-c_{i}\right|=\left|x_{p}-z_{p}\right|$, by (i) of Claim 1;

2) $\left|x_{p}-M_{q} z_{p}-c_{q}\right|=\left|x_{p}-M_{i} z_{p}-c_{q}+c_{i}-c_{i}\right|=$

$$
=\left|x_{p}-z_{p}+c_{i}-c_{q}\right| \text {, where } c_{i}-c_{q}=\frac{2 F_{i}}{\left|F_{i}\right|}\left(\frac{1}{\left|F_{i}\right|}+\frac{1}{\left|F_{q}\right|}\right) \text {; }
$$

3) $\frac{F_{i}^{T}}{\left|F_{i}\right|}\left(z_{p}-x_{p}\right)=\frac{F_{i}^{T}}{\left|F_{i}\right|}\left(z_{p}-s_{\circ}-x_{p}+s_{\circ}\right)=$

$$
=\frac{F_{i}^{T}}{\left|F_{i}\right|}\left(z_{p}-s_{\circ}\right)+\frac{F_{q}^{T}}{\left|F_{q}\right|}\left(x_{p}-s_{\circ}\right) \leq\left(\frac{1}{\left|F_{i}\right|}+\frac{1}{\left|F_{q}\right|}\right) \text {. }
$$

Therefore,

$$
\begin{aligned}
& \left|x_{p}-M_{i} z_{p}-c_{i}\right|^{2}-\left|x_{p}-M_{q} z_{p}-c_{q}\right|^{2}= \\
& \quad=\left|x_{p}-z_{p}\right|^{2}-\left|x_{p}-z_{p}+c_{i}-c_{q}\right|^{2} \\
& \quad=\left(\frac{F_{i}^{T}}{\left|F_{i}\right|}\left(x_{p}-z_{p}\right)\right)^{2}-\left(\frac{F_{i}^{T}}{\left|F_{i}\right|}\left(x_{p}-z_{p}+c_{i}-c_{q}\right)\right)^{2} \\
& \quad=-2\left(x_{p}-z_{p}\right)^{T} \frac{F_{i} F_{i}^{T}}{\left|F_{i}\right|^{2}}\left(c_{i}-c_{q}\right)-\left(\frac{F_{i}^{T}}{\left|F_{i}\right|}\left(c_{i}-c_{q}\right)\right)^{2} \\
& \quad=4\left(z_{p}-x_{p}\right)^{T} \frac{F_{i}}{\left|F_{i}\right|}\left(\frac{1}{\left|F_{i}\right|}+\frac{1}{\left|F_{q}\right|}\right)-4\left(\frac{1}{\left|F_{i}\right|}+\frac{1}{\left|F_{q}\right|}\right)^{2} \\
& \quad \leq 4\left(\frac{1}{\left|F_{i}\right|}+\frac{1}{\left|F_{q}\right|}\right)^{2}-4\left(\frac{1}{\left|F_{i}\right|}+\frac{1}{\left|F_{q}\right|}\right)^{2}=0 .
\end{aligned}
$$

Finally, consider an impact of $\mathcal{X}$, then

$$
\begin{aligned}
\left|x^{+}-m_{q^{+}}\left(z^{+}\right)\right|_{\bar{P}} & =\left|m_{i}(x)-m_{\delta(q, i)}(z)\right|_{\bar{P}} \\
& =\left|m_{i}(x)-z\right|_{\bar{P}} \\
& =\left|x-m_{i}(z)\right|_{\bar{P}} \\
& \leq\left|x-m_{q}(z)\right|_{\bar{P}}
\end{aligned}
$$

where the last identity follows from (i) of Claim 2, and the last inequality follows from (49).

Proposition 6 (Two orthogonal walls): Consider $F_{1}, F_{2} \in \mathbb{R}^{2}$ such that $F_{1}^{T} F_{2}=0, \overline{\mathcal{I}}:=\{0,1,2\}$, and define $\mathcal{Q}:=\overline{\mathcal{I}} \cup\{3\}, M_{3}:=M_{1} M_{2}, c_{3}:=M_{1} c_{2}+c_{1}$, so that $m_{3}(z)=m_{1}\left(m_{2}(z)\right)$. Then, under Assumption 1, Assumption 5 is satisfied and (46) is satisfied for the function $\delta$ in Figure 5.

\begin{tabular}{c|cc}
$\mathbf{q} \backslash \mathbf{i}$ & 1 & 2 \\
\hline 0 & 1 & 2 \\
1 & 0 & 3 \\
2 & 3 & 0 \\
3 & 2 & 1
\end{tabular}

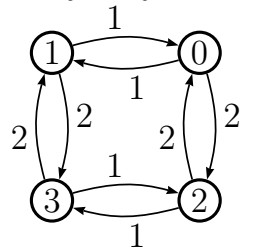

Figure 5. Definition of $\delta$ for two orthogonal walls.

The proof of Proposition 6 is based on the following lemma.

Lemma 2: $M_{1} M_{2}=M_{2} M_{1}=-I, M_{1} c_{2}+c_{1}=M_{2} c_{1}+c_{2}$, and $m_{1}\left(m_{2}(s)\right)=m_{2}\left(m_{1}(s)\right)$, for each $s \in \mathbb{R}^{4}$. Moreover, for each $s \in \mathbb{R}^{4}, m_{3}\left(m_{1}(s)\right)=m_{2}(s), m_{3}\left(m_{2}(s)\right)=m_{1}(s), m_{1}\left(m_{3}(s)\right)=m_{2}(s)$, $m_{2}\left(m_{3}(s)\right)=m_{1}(s)$. 
Proof:

$$
\begin{aligned}
M_{1} M_{2} & =R\left(F_{1}\right)^{T}\left[\begin{array}{cc}
1 & 0 \\
0 & -1
\end{array}\right] R\left(F_{1}\right) R\left(F_{2}\right)^{T}\left[\begin{array}{cc}
1 & 0 \\
0 & -1
\end{array}\right] R\left(F_{2}\right) \\
& =-R\left(F_{1}\right)^{T} R\left(F_{1}\right) R\left(F_{2}\right)^{T} R\left(F_{2}\right) \\
& =-I \\
& =-R\left(F_{2}\right)^{T} R\left(F_{2}\right) R\left(F_{1}\right)^{T} R\left(F_{1}\right) \\
& =R\left(F_{2}\right)^{T}\left[\begin{array}{cc}
1 & 0 \\
0 & -1
\end{array}\right] R\left(F_{2}\right)^{T} R\left(F_{1}\right)^{T}\left[\begin{array}{cc}
1 & 0 \\
0 & -1
\end{array}\right] R\left(F_{1}\right)^{T} \\
& =M_{2} M_{1} .
\end{aligned}
$$

From (51), we also have that $M_{1}=-M_{2}$, since $M_{1} M_{1}=I$. Using this fact, the fact that $F_{1} c_{2}=F_{2} c_{1}=0$, and (iii) of Claim 1, consider the basis given by $\left\{\frac{F_{1}^{T}}{\left|F_{1}\right|}, \frac{F_{2}^{T}}{\left|F_{2}\right|}\right\}$, and assume $i \in\{1,2\}$ and $j \in\{1,2\}, j \neq i$, then

$$
\begin{aligned}
& \frac{F_{i}^{T}}{\left|F_{i}\right|}\left(M_{i} c_{j}+c_{i}\right)=\frac{F_{i}^{T}}{\left|F_{i}\right|}\left(-c_{j}+c_{i}\right)=\frac{F_{i}^{T}}{\left|F_{i}\right|} c_{i}= \\
& =-\frac{F_{i}^{T}}{\left|F_{i}\right|} M_{i} c_{i}=\frac{F_{i}^{T}}{\left|F_{i}\right|} M_{j} c_{i}=\frac{F_{i}^{T}}{\left|F_{i}\right|}\left(M_{j} c_{i}+c_{j}\right) .
\end{aligned}
$$

Thus, the identity $m_{1}\left(m_{2}(s)\right)=m_{2}\left(m_{1}(s)\right)$ follows from (51) and (52). Finally, the identities on $m_{3}(s)$ follow from the definition of $m_{3}(s), m_{1}\left(m_{2}(s)\right)=m_{2}\left(m_{1}(s)\right)$ and (v) of Claim 1.

Proof of Proposition 6. Suppose $\left|x-m_{3}(z)\right|=0$ with $x, z \in \mathcal{F}$. Then $F_{i}\left(x_{p}-s_{\circ}\right) \leq 1, i \in\{1,2\}$, which implies $F_{i}^{T}\left(M_{i}\left(M_{j} z_{p}+c_{2}\right)+c_{1}-s_{\circ}\right) \leq 1$, where $j \in\{1,2\}, j \neq i$. Moreover, we have

$$
\begin{aligned}
F_{i}^{T} & \left(M_{i}\left(M_{j} z_{p}+c_{j}\right)+c_{i}-s_{\circ}\right)= \\
& =F_{i}^{T}\left(-\left(M_{j} z_{p}+c_{j}\right)+c_{i}-s_{\circ}\right) \\
& =F_{i}^{T}\left(M_{i} z_{p}+c_{i}-s_{\circ}\right) \\
& =F_{i}^{T}\left(-z_{p}+c_{i}-s_{\circ}\right) \\
& =-F_{i}^{T}\left(z_{p}-s_{\circ}\right)-2 F_{i}^{T} s_{\circ}+F_{i}^{T} c_{i} \\
& =-F_{i}^{T}\left(z_{p}-s_{\circ}\right)-2 F_{i}^{T} s_{\circ}+2\left(1+F_{i}^{T} s_{\circ}\right) \\
& =-F_{i}^{T}\left(z_{p}-s_{\circ}\right)+2 \geq 1,
\end{aligned}
$$

therefore $F_{i}\left(x_{p}-s_{\circ}\right)=F_{i}\left(z_{p}-s_{\circ}\right)=1$. Looking at the velocity vector, $F_{i}^{T} x_{v}=F_{i}^{T} M_{i} M_{j} z_{v}=$ $-F_{i}^{T} M_{j} z_{v}=-F_{i}^{T} z_{v}$, since $M_{j}$ does not modify the component of $z_{v}$ normal to $F_{i}$. Thus, either $x$ or $z$ impacts on the $i$ th wall. In a similar way, $F_{j} x_{v}=F_{j} M_{i} M_{j} z_{v}=F_{j} M_{j} M_{i} z_{v}=-F_{j} M_{i} z_{v}=F_{j} z_{v}$, thus either $x$ or $z$ impacts on the $j$ th wall. It follows that either $\mathcal{M}(x)=\{1,2\}$ or $\mathcal{M}(z)=\{1,2\}$, which is forbidden by Assumption 1. This proves Assumption 5 .

To establish (46) we analyze the cases (cl) $q \in\{1,2\}, i \in\{1,2\}, i \neq q$, and (c2) $q=3, i \in\{1,2\}$, since the remaining cases have been developed in the previous sections, for the one-wall case. For $(c 1)$, consider a jump of $\mathcal{Z}$, then

$$
\begin{aligned}
\left|x^{+}-m_{q^{+}}\left(z^{+}\right)\right|_{\bar{P}} & =\left|x-m_{\delta(q, i)}\left(m_{i}(z)\right)\right|_{\bar{P}} \\
& =\left|x-m_{3}\left(m_{i}(z)\right)\right|_{\bar{P}} \\
& =\left|x-m_{q}(z)\right|_{\bar{P}},
\end{aligned}
$$

where the last identity follows from Lemma 2. For a jump of $\mathcal{X}$ the analysis above can be repeated, by using $\left|m_{i}(x)-m_{\delta(q, i)}(z)\right|_{\bar{P}}=\left|x-m_{i}\left(m_{\delta(q, i)}(z)\right)\right|_{\bar{P}}$ and Lemma 2. For $(c 2)$, consider a jump of $\mathcal{Z}$, and note that if $i \in\{1,2\}$ and $q=3$ then $\delta(q, i)=j \in\{1,2\}, j \neq i$, thus $m_{\delta(q, i)}\left(m_{i}(z)\right)=m_{j}\left(m_{i}(z)\right)=m_{3}(z)$. Then,

$$
\left|x^{+}-m_{q^{+}}\left(z^{+}\right)\right|_{\bar{P}}=\left|x-m_{\delta(q, i)}\left(m_{i}(z)\right)\right|_{\bar{P}}=\left|x-m_{q}(z)\right|_{\bar{P}},
$$

The analysis of a jump of $\mathcal{X}$ follows similar steps.

By combining parallel and orthogonal walls, we can characterize sufficient conditions for rectangular billiards.

Proposition 7 (Rectangles): Consider walls $F_{i}, i \in \mathcal{I}:=\{1,2,3,4\}$ such that $F_{1}^{T} F_{2}=0, F_{1}^{T} F_{4}=0$, $\frac{F_{1}}{\left|F_{1}\right|}=-\frac{F_{3}}{\left|F_{3}\right|}$, and $\frac{F_{2}}{\left|F_{2}\right|}=-\frac{F_{4}}{\left|F_{4}\right|}$. Consider $\overline{\mathcal{I}}=\mathcal{I} \cup\{0\}$, define $\mathcal{Q}=\overline{\mathcal{I}} \cup\{5,6,7,8\}$ and assume that $P>0$ is a diagonal matrix. Define also $M_{5}:=M_{1} M_{2}, M_{6}:=M_{2} M_{3}, M_{7}:=M_{3} M_{4}, M_{8}:=M_{4} M_{1}$, 


\begin{tabular}{c|cccc}
$\mathbf{q} \backslash \mathbf{i}$ & 1 & 2 & 3 & 4 \\
\hline 0 & 1 & 2 & 3 & 4 \\
1 & 0 & 5 & 0 & 8 \\
2 & 5 & 0 & 6 & 0 \\
3 & 0 & 6 & 0 & 7 \\
4 & 8 & 0 & 7 & 0 \\
5 & 2 & 1 & 2 & 1 \\
6 & 2 & 3 & 2 & 3 \\
7 & 4 & 3 & 4 & 3 \\
8 & 4 & 1 & 4 & 1
\end{tabular}
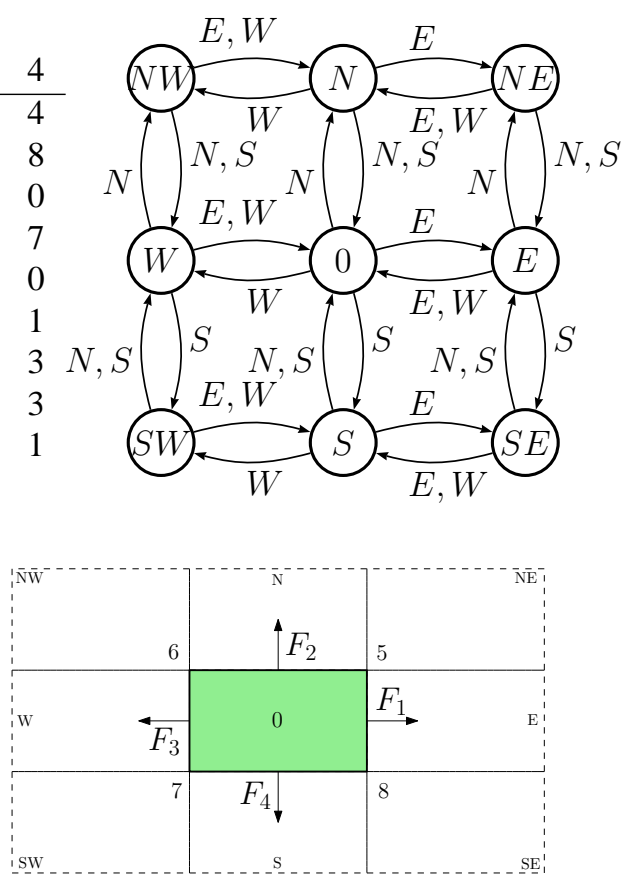

Figure 6. Definition of the update function $\delta$ for a rectangular billiard. For simplicity, we used an intuitive notation in the graph representation of $\delta$ and on the billiard representation, in which the four walls $\{1,2,3,4\}$ are denoted respectively as East, North, West, and South walls, while the extra mirroring functions, given by $q \in\{5,6,7,8\}$ are denoted respectively as NE, NW, SW, and SE. Note the iterative application of the results for parallel and orthogonal walls

and $c_{5}:=M_{1} c_{2}+c_{1}, c_{6}:=M_{2} c_{3}+c_{2}, c_{7}:=M_{3} c_{4}+c_{3}, c_{8}:=M_{4} c_{1}+c_{4}$. Then, under Assumption 1, Assumption 5 is satisfied, and (46) is satisfied for $\delta$ given in Figure 6.

Proof: From the hypotheses of Proposition 7, $m_{5}(s)=m_{1}\left(m_{2}(s)\right), m_{6}(s)=m_{2}\left(m_{3}(s)\right), m_{7}(s)=$ $m_{3}\left(m_{4}(s)\right)$, and $m_{8}(s)=m_{4}\left(m_{1}(s)\right)$, for all $s \in \mathbb{R}^{4}$. Thus, Assumption 5 can be established by considering the analysis developed in the proof of Proposition 6. Moreover, looking at the automaton in Figure 6, note that $\delta$ is constructed by combining Propositions 5 and 6 , thus the analysis can be developed by following the arguments of those propositions. Cases not analyzed directly in Propositions 5 and 6 arise from the configuration $q \in\{5,6,7,8\}$ for impacts on walls $3,4,1,2$, respectively. The proof, in these cases, follows the approach of the two-parallel walls case, from which we have that $\left|x-m_{3}\left(m_{5}(z)\right)\right|_{\bar{P}}=\left|x-m_{3}\left(m_{1}\left(m_{2}(z)\right)\right)\right|_{\bar{P}} \leq\left|x-m_{2}(z)\right|_{\bar{P}},\left|x-m_{4}\left(m_{6}(z)\right)\right|_{\bar{P}}=\left|x-m_{4}\left(m_{2}\left(m_{3}(z)\right)\right)\right|_{\bar{P}} \leq$ $\left|x-m_{3}(z)\right|_{\bar{P}},\left|x-m_{1}\left(m_{7}(z)\right)\right|_{\bar{P}}=\left|x-m_{1}\left(m_{3}\left(m_{4}(z)\right)\right)\right|_{\bar{P}} \leq\left|x-m_{4}(z)\right|_{\bar{P}}$, and $\left|x-m_{2}\left(m_{8}(z)\right)\right|_{\bar{P}}=$ $\left|x-m_{2}\left(m_{4}\left(m_{1}(z)\right)\right)\right|_{\bar{P}} \leq\left|x-m_{1}(z)\right|_{\bar{P}}$.

Remark 12: Further results on sufficient conditions for Theorems 4 and 5 can be established for two walls that meet at special acute angles and for equilateral triangles, as documented in [8, Section VI.F] and [8, Section VI.G].

Remark 13: Propositions 5-7 characterize a selection policy among mirrored targets and real target based on a specific function $\delta$, whose definition strictly depends on the billiard shape. Differently from this approach, tracking in [8] is characterized by a Lyapunov-based selection policy between mirrored and real targets, which is implemented by enforcing updates for $q$ that satisfy $q^{+}=\operatorname{argmin}_{i \in \mathcal{Q}}\left|x^{+}-m_{i}\left(z^{+}\right)\right|_{\bar{P}}$, where $\mathcal{Q}$ and each $m_{i}(s), i \in \mathcal{Q}$, depend on the particular billiard shape, and where $x^{+}$and $z^{+}$denote the reset of $x$ and $z$ given by an impact on some wall of the billiard. These two approaches are connected to each other through the function $\delta$, which can be interpreted at impacts as the explicit solution of the minimization problem, that is, if the initial condition $q(0,0)=\operatorname{argmin}_{j \in \mathcal{Q}}\left|x(0,0)-m_{j}(z(0,0))\right|$ then at each impact $\delta(q, i)=\operatorname{argmin}_{j \in \mathcal{Q}}\left|x^{+}-m_{j}\left(z^{+}\right)\right|$, where either $\left(x^{+}, z^{+}\right)=\left(m_{i}(x), z\right)$ or $\left(x^{+}, z^{+}\right)=$ $\left(x, m_{i}(z)\right), i \in \mathcal{I}$.

Finally, for the billiards considered in this section we can extend Proposition 4 as follows, to provide a parallel to Proposition 1. 
Proposition 8: For billiards defined by parallel walls, orthogonal walls and rectangles, $\left|x_{p}-z_{p}\right| \leq$ $\left|x-m_{q}(z)\right|$ for each $X=(x, z, \sigma, q) \in \overline{\mathcal{C}} \cup \overline{\mathcal{D}}$.

Proof: (Sketch) The case of parallel walls follows from Proposition 1 since there no extra $q$ are introduced. The proof for rectangular billiards is similar to that of the orthogonal walls case. For two orthogonal walls we have $\overline{\mathcal{I}}=\{0,1,2\}$ and $\mathcal{Q}=\{3\} \cup \overline{\mathcal{I}}$. Consider the line $\ell$ that connects $M_{1} z_{p}+c_{1}$ to $M_{1} M_{2} z_{p}+M_{1} c_{2}+c_{1}=M_{3} z_{p}+c_{3}$ and note that $\ell$ is orthogonal to $F_{2}$ (a similar argument can be developed for the line connecting $M_{2} z_{p}+c_{2}$ and $M_{3} z_{p}+c_{3}$ which is orthogonal to $F_{1}$ ). Then, following Proposition 1, define $\mathcal{S}_{\ell}:=\left\{s \in \mathbb{R}^{2} \mid s=\lambda\left(M_{1} z_{p}+c_{1}\right)+(1-\lambda)\left(M_{3} z_{p}+c_{3}\right)\right\}, \eta:=\operatorname{argmin}\left|x_{p}-s\right|$, and $\mathcal{S}_{F_{2}}:=\left\{s \in \mathbb{R}^{2} \mid F_{2}^{T}\left(s-s_{0}\right)=1\right\}$. For these sets we have $\left|M_{1} z_{p}+c_{1}\right|_{\mathcal{S}_{F_{2}}}=\left|M_{3} z_{p}+c_{3}\right| \mathcal{S}_{F_{2}}$, and $\left|x_{p}-M_{3} z_{p}-c_{3}\right|^{2}=\left|x_{p}\right|_{\mathcal{S}_{\ell}}^{2}+\left|\eta-M_{3} z_{p}-c_{3}\right|^{2} \geq\left|x_{p}\right|_{\mathcal{S}_{\ell}}^{2}+\left|\eta-M_{1} z_{p}-c_{1}\right|^{2}=\left|x_{p}-M_{1} z_{p}-c_{1}\right|^{2}$, where the second inequality follows from $F_{2}^{T}\left(\eta-s_{\circ}\right) \leq 1$, since $F_{2}^{T}\left(x_{p}-s_{\circ}\right) \leq 1$. Following a similar argument for $M_{2} z_{p}+c_{2}$ and $M_{3} z_{p}+c_{3}$, we can establish that $\left|x-m_{3}(z)\right| \geq\left|x_{p}-M_{3} z_{p}-c_{3}\right| \geq$ $\max \left(\left|x_{p}-M_{1} z_{p}-c_{1}\right|,\left|x_{p}-M_{2} z_{p}-c_{2}\right|\right) \geq\left|x_{p}-z_{p}\right|$, where the last inequality follows from Proposition 1.

\section{OUTPUT FEEDBACK TRACKING}

Henceforth, we combine the tracking and state estimation algorithms of the previous section to construct an output feedback controller. We consider the following setup: $\mathcal{Z}$ is the exogenous system and we have full access to its state, $\mathcal{X}$ is the controlled system (the plant) and we measure its position $y=C x$, and $\widehat{\mathcal{X}}$ is the dynamic controller whose output drives $\mathcal{X}$ to achieve asymptotic tracking of $\mathcal{Z}$. We assume to know the wall impacted by each system.

The exposition of the dynamic controller uses notation and results from the previous sections, thus it is based on the quantities $M_{i}, c_{i}$ and $m_{i}(s), i \in \mathcal{Q} \subset \mathbb{N}, \mathcal{Q} \supset \overline{\mathcal{I}}$, defined in (38) for $i \in \overline{\mathcal{I}}$, and in (39) for $i \in \mathcal{Q} \backslash \overline{\mathcal{I}}$. The continuous (flow) dynamics of the closed-loop system is given by

$$
\begin{aligned}
& \mathcal{Z}:\left\{\dot{z}=\bar{A} z+\bar{B} d_{1}\right. \\
& \mathcal{X}:\left\{\begin{array}{l}
\dot{x}=\bar{A} x+\bar{B}\left(d_{2}+u_{c}\right) \\
y=C x
\end{array}\right. \\
& \widehat{\mathcal{X}}:\left\{\begin{aligned}
\dot{\hat{x}} & =\bar{A} \hat{x}+u_{o} \\
\dot{q} & =0 \\
\dot{\hat{q}} & =0,
\end{aligned}\right.
\end{aligned}
$$

and by (7a), where, for simplicity of notation, $d_{1}$ and $d_{2}$ are signals measured by the dynamic controller, possibly replacing functions like $\alpha$ in (3) or (30) and $\phi$ in (8), used in previous sections. Generalizing the results of the previous sections, $u_{c}$ and $u_{o}$ are defined by

$$
\begin{aligned}
& u_{c}=M_{q} d_{1}+\bar{K}\left(m_{\hat{q}}(\hat{x})-m_{q}(z)\right) \\
& u_{o}=\bar{M}_{\hat{q}} \bar{B}\left(d_{2}+u_{c}\right)+\bar{L}\left[C \hat{x}-C m_{\hat{q}}(x)\right]
\end{aligned}
$$

where $C m_{\hat{q}}(x)=M_{\hat{q}} y+c_{\hat{q}}$, and $\bar{K} \in \mathbb{R}^{2 \times 4}$ and $\bar{L} \in \mathbb{R}^{4 \times 2}$ are respectively the controller and the observer gains. The overall state is defined as $X=(z, x, \hat{x}, q, \hat{q}, \sigma)$ and the flow set is given by

$$
\overline{\mathcal{C}}:=\mathcal{K} \times \mathcal{F} \times \mathcal{F} \times \mathcal{Q} \times \mathcal{Q} \times[0, N],
$$

where $\mathcal{Q} \in \mathbb{N}, \mathcal{Q} \supseteq \overline{\mathcal{I}}$, characterizes the set where the two automata with states $q$ and $\hat{q}$ take values. 
The discrete (jump) dynamics is given by

$$
\left\{\begin{array}{l}
z^{+} \in \bigcup_{i \in \mathcal{M}(z)} m\left(F_{i}, z\right) \\
x^{+}=x \\
\hat{x}^{+}=\hat{x} \\
q^{+}=\delta(q, i) \\
\hat{q}^{+}=\hat{q} \\
\sigma^{+}=\sigma-1
\end{array} ;\left\{\begin{array}{l}
z^{+}=z \\
x^{+} \in \bigcup_{i \in \mathcal{M}(x)} m\left(F_{i}, x\right) \\
\hat{x}^{+}=\hat{x} \\
q^{+}=\delta(q, i) \\
\hat{q}^{+}=\delta(\hat{q}, i) \\
\sigma^{+}=\sigma-1
\end{array} ;\left\{\begin{array}{l}
z^{+}=z \\
x^{+}=x \\
\hat{x}^{+} \in \bigcup_{i \in \mathcal{M}(\hat{x})} m\left(F_{i}, \hat{x}\right) \\
q^{+}=q \\
\hat{q}^{+}=\delta(\hat{q}, i) \\
\sigma^{+}=\sigma-1
\end{array}\right.\right.\right.
$$

respectively for $X \in \overline{\mathcal{D}}_{z}, X \in \overline{\mathcal{D}}_{x}$, and $X \in \overline{\mathcal{D}}_{\hat{x}}$, with those sets defined as

$$
\begin{aligned}
\overline{\mathcal{D}}_{z} & :=\{X \in \overline{\mathcal{C}} \mid z \in \mathcal{J}, \sigma \in[1, N]\}, \\
\overline{\mathcal{D}}_{x} & :=\{X \in \overline{\mathcal{C}} \mid x \in \mathcal{J}, \sigma \in[1, N]\}, \\
\overline{\mathcal{D}}_{\hat{x}} & :=\left\{X \in \overline{\mathcal{C}} \mid\left(\hat{x}_{p}, \hat{x}_{v}+u_{o, p}\right) \in \mathcal{J}, \sigma \in[1, N]\right\}, \\
\overline{\mathcal{D}} & :=\overline{\mathcal{D}}_{z} \cup \overline{\mathcal{D}}_{x} \cup \overline{\mathcal{D}}_{\hat{x}} .
\end{aligned}
$$

Similar to (16b), in the four different intersections among $\overline{\mathcal{D}}_{z}, \overline{\mathcal{D}}_{x}, \overline{\mathcal{D}}_{\hat{x}}$, the jump rule is the union of the corresponding jump rules in (59). This definition produces an outer semicontinuous set-valued jump map, thereby guaranteeing robustness (see [12]). The function $\delta$ in (59) depends on the billiard shape, as discussed in the previous sections, and the vector $u_{o, p}$ used in the definition of the jump set above is given by $u_{o}=\left[u_{o, p}^{T} \mid u_{o, v}^{T}\right]^{T}$ (this follows from the observer construction in Section IV). Note that $q$ is updated when either $\mathcal{Z}$ or $\mathcal{X}$ jumps, following the approach of Section III ( $\mathcal{X}$ tracks $\mathcal{Z})$, while $\hat{q}$ is updated when either $\mathcal{X}$ or $\widehat{\mathcal{X}}$ jumps, following Section IV (note that $\mathcal{X}$ plays here the role of the exogenous system of Section IV whose state is estimated by the observer $\widehat{\mathcal{X}}$ ).

The next stability result is based on the following two assumptions which extend to the output feedback case the assumptions of the previous sections.

Assumption 6: The exosystem $\mathcal{Z}$ is restricted to a compact set $\mathcal{K}$ which satisfies Assumption $1 ; K$ satisfies Assumption 2 and $\bar{K}:=K \otimes I_{2} ; L$ satisfies Assumption 4 and $\bar{L}:=L \otimes I_{2}$.

Assumption 7: Given the compact set

$$
\begin{array}{r}
\mathcal{A}:=\left\{(z, x, \hat{x}, q, \hat{q}, \sigma) \in \mathcal{K} \times \mathbb{R}^{4} \times \mathbb{R}^{4} \times \mathcal{Q} \times \mathcal{Q} \times[0, N] \mid\right. \\
\left.x=m_{q}(z), \hat{x}=m_{\hat{q}}(x)\right\},
\end{array}
$$

for all $X=(z, x, \hat{x}, q, \hat{q}, \sigma) \in \mathcal{A}$, if $X \in \overline{\mathcal{C}} \cup \overline{\mathcal{D}}$ then $q, \hat{q} \in \overline{\mathcal{I}}$.

Using the argument of Proposition 4, it is possible to show that when $X \in \mathcal{A}$ then $z_{p}=x_{p}=\hat{x}_{p}$ and that $z=x=\hat{x}$ for each $X \in \mathcal{A} \backslash \overline{\mathcal{D}}$. Moreover, the following bounds hold.

Proposition 9: Under Assumption 7, for $\mathcal{A}$ in (61), for each $X=(z, x, \hat{x}, q, \hat{q}, \sigma) \in \overline{\mathcal{C}} \cup \overline{\mathcal{D}}$,

$$
\begin{aligned}
\frac{1}{\sqrt{6 r}}\left(\left|x-m_{q}(z)\right|+\left|\hat{x}-m_{\hat{q}}(z)\right|\right) & \leq|X|_{\mathcal{A}} \\
\sqrt{2}\left(\left|x-m_{q}(z)\right|+\left|\hat{x}-m_{\hat{q}}(z)\right|\right) & \geq|X|_{\mathcal{A}}
\end{aligned}
$$

where

$$
\begin{aligned}
r & :=\max \left(r_{1}, r_{2}\right), \\
r_{1} & :=\max _{z \in \mathcal{K}, s_{1}, s_{2} \in \mathcal{Q}}\left\{1,\left|m_{s_{1}}(z)-m_{s_{2}}(z)\right|\right\}, \\
r_{2} & :=\max _{z \in \mathcal{K}, s_{1}, s_{2}, s_{3} \in \mathcal{Q}}\left\{1,\left|m_{s_{1}}\left(m_{s_{3}}(z)\right)-m_{s_{2}}\left(m_{s_{3}}(z)\right)\right|\right\} .
\end{aligned}
$$

Proof: We use $(i)$ and (ii) in the proof of Proposition 1. For the last inequality in (62),

$$
\begin{aligned}
|X|_{\mathcal{A}}^{2} & =\min _{a \in \mathcal{K}, \beta, \hat{\beta} \in \mathcal{Q}}\left(|z-a|^{2}+\left|x-m_{\beta}(a)\right|^{2}+\left|\hat{x}-m_{\hat{\beta}}\left(m_{\beta}(a)\right)\right|^{2}+\right. \\
& \left.\quad+|q-\beta|^{2}+|\hat{q}-\hat{\beta}|^{2}\right) \\
& \leq\left|x-m_{q}(z)\right|^{2}+\left|\hat{x}-m_{\hat{q}}\left(m_{q}(z)\right)\right|^{2} \\
& \leq\left|x-m_{q}(z)\right|^{2}+\left|\hat{x}-m_{\hat{q}}(x)\right|^{2}+\left|m_{\hat{q}}(x)-m_{\hat{q}}\left(m_{q}(z)\right)\right|^{2} \\
& \leq\left|x-m_{q}(z)\right|^{2}+\left|\hat{x}-m_{\hat{q}}(x)\right|^{2}+\left|x-m_{q}(z)\right|^{2} \\
& \leq 2\left(\left|x-m_{q}(z)\right|+\left|\hat{x}-m_{\hat{q}}(x)\right|\right)^{2} .
\end{aligned}
$$


For the first inequality in (62), consider the following two facts which will be proven next, in (65) and (66): $\left(a_{1}\right)|X|_{\mathcal{A}} \geq \frac{1}{r}\left|x-m_{q}(z)\right|$ and $\left(a_{2}\right)|X|_{\mathcal{A}} \geq \frac{1}{r}\left|\hat{x}-m_{\hat{q}}\left(m_{q}(z)\right)\right|$. Then,

$$
\begin{aligned}
|X|_{\mathcal{A}}^{2} & \geq \frac{1}{3 r^{2}}\left(2\left|x-m_{q}(z)\right|^{2}+\left|\hat{x}-m_{\hat{q}}\left(m_{q}(z)\right)\right|^{2}\right) \\
& =\frac{1}{3 r^{2}}\left(\left|x-m_{q}(z)\right|^{2}+\left|m_{\hat{q}}(x)-m_{\hat{q}}\left(m_{q}(z)\right)\right|^{2}+\right. \\
& \left.+\left|\hat{x}-m_{\hat{q}}\left(m_{q}(z)\right)\right|^{2}\right) \\
& \geq \frac{1}{3 r^{2}}\left(\left|x-m_{q}(z)\right|^{2}+\left|\hat{x}-m_{\hat{q}}(x)\right|^{2}\right) \\
& \geq \frac{1}{6 r^{2}}\left(\left|x-m_{q}(z)\right|+\left|\hat{x}-m_{\hat{q}}(x)\right|\right)^{2}
\end{aligned}
$$

where the last inequality follows from $|a|^{2}+|b|^{2} \geq 2|a||b|$, from which $|a|^{2}+|b|^{2} \geq \frac{1}{2}(|a|+|b|)^{2}$.

Considering the proof of Propositions 1 and 4, to prove $\left(a_{1}\right)$ we can reason as follows

$$
\begin{aligned}
|X|_{\mathcal{A}}^{2} & \geq \min _{a \in \mathcal{K}, \beta \in \mathcal{Q}}|z-a|^{2}+\left|x-m_{\beta}(a)\right|^{2}+|q-\beta|^{2} \\
& \geq \frac{1}{r_{1}^{2}}\left|x-m_{q}(z)\right|^{2} \geq \frac{1}{r^{2}}\left|x-m_{q}(z)\right|^{2} .
\end{aligned}
$$

In a similar way, for $\left(a_{2}\right)$ we use the identity $r_{1}=\max _{z \in \mathcal{K}, \beta, q, \hat{q} \in \mathcal{Q}}\left|m_{\hat{q}}\left(m_{\beta}(z)\right)-m_{\hat{q}}\left(m_{q}(z)\right)\right|$, getting

$$
\begin{aligned}
|X|_{\mathcal{A}}^{2} & \geq \min _{a \in \mathcal{K}, \beta, \hat{\beta} \in \mathcal{Q}}\left(|z-a|^{2}+\left|\hat{x}-m_{\hat{\beta}}\left(m_{\beta}(a)\right)\right|^{2}+\right. \\
& \left.+|\hat{q}-\hat{\beta}|^{2}+|q-\beta|^{2}\right) \\
& =\min _{a \in \mathcal{K}, \beta, \hat{\beta} \in \mathcal{Q}}\left(\left|m_{\hat{\beta}}\left(m_{\beta}(z)\right)-m_{\hat{\beta}}\left(m_{\beta}(a)\right)\right|^{2}+\right. \\
& \left.\geq \min _{\beta, \hat{\beta} \in \mathcal{Q}}\left|\hat{x}-m_{\hat{\beta}}\left(m_{\beta}(a)\right)\right|^{2}+|\hat{q}-\hat{\beta}|^{2}+|q-\beta|^{2}\right) \\
& \geq \min _{\beta \in \mathcal{Q}} \frac{1}{r_{2}^{2}}\left|\hat{x}-m_{\hat{q}}\left(m_{\beta}(z)\right)\right|^{2}+|\hat{q}-\hat{\beta}|^{2}+|q-\beta|^{2} \\
& \geq \min _{\beta \in \mathcal{Q}} \frac{1}{r_{2}^{2}}\left|\hat{x}-m_{\hat{q}}\left(m_{\beta}(z)\right)\right|^{2}+ \\
& +\frac{1}{r_{1}^{2}}\left|m_{\hat{q}}\left(m_{\beta}(z)\right)-m_{\hat{q}}\left(m_{q}(z)\right)\right|^{2} \\
& \geq \frac{1}{r^{2}}\left|\hat{x}-m_{\hat{q}}\left(m_{q}(z)\right)\right|^{2} .
\end{aligned}
$$

We can now state the exponential stability of the set $\mathcal{A}$ in (61).

Theorem 6: Under Assumptions 6 and 7, consider a pair $\left(P_{c}, H_{c}\right)$ which satisfies (25) for $A_{c l}=\left[\begin{array}{cc}0 & 1 \\ k_{1} & k_{2}\end{array}\right]$, a pair $\left(P_{c}, H_{c}\right)$ which satisfy (25) for $A_{c l}=\left[\begin{array}{ll}\ell_{1} & 1 \\ \ell_{2} & 0\end{array}\right]$, and define $\bar{P}_{c}=P_{c} \otimes I_{2}, \bar{P}_{o}=P_{o} \otimes I_{2}$. If for each $X \in \overline{\mathcal{D}}$,

$$
\begin{aligned}
\left|x^{+}-m_{q^{+}}\left(z^{+}\right)\right|_{\bar{P}_{c}} \leq\left|x-m_{q}(z)\right|_{\bar{P}_{c}} \\
\left|\hat{x}^{+}-m_{\hat{q}^{+}}\left(x^{+}\right)\right|_{\bar{P}_{o}} \leq\left|\hat{x}-m_{\hat{q}}(x)\right|_{\bar{P}_{o}},
\end{aligned}
$$

then, for each $\rho>0$ and $N>0, \mathcal{A}$ in (61) is GES for the closed-loop system.

Proof: Define $e_{1}:=\hat{x}-m_{\hat{q}}(x)$ and $e_{2}:=x-m_{q}(z)$. During flows, using the identity $\bar{M}_{\hat{q}} \bar{A} x=$ $\overline{A M}_{\hat{q}} x=\bar{A} m_{\hat{q}}(x)$ (see the proof of Proposition 2), we have

$$
\begin{aligned}
\dot{e}_{1} & =\bar{A} \hat{x}+\bar{M}_{\hat{q}} \bar{B} u_{c}+\bar{L} C\left[\hat{x}-\bar{m}_{\hat{q}}(x)\right]-\bar{M}_{\hat{q}}\left(\bar{A} x+\bar{B} u_{c}\right) \\
& =(\bar{A}+\bar{L} C)\left(\hat{x}-m_{\hat{q}}(x)\right)=(\bar{A}+\bar{L} C) e_{1},
\end{aligned}
$$

and using the identities $\bar{B} M_{q} d_{1}=\bar{M}_{q} \bar{B} d_{1}, \bar{M}_{q} \bar{A} z=\bar{A} m_{q}(z)$, and $\bar{M}_{\hat{q}} \bar{c}(\hat{q})+\bar{c}(\hat{q})=0$, we have

$$
\begin{aligned}
\dot{e}_{2}= & \bar{A} x+\bar{B} M_{q} d_{1}+\overline{B K}\left(m_{\hat{q}}(\hat{x})-m_{q}(z)\right)+ \\
& -\bar{M}_{q}\left(\bar{A} z+\bar{B} d_{1}\right) \\
= & \bar{A}\left(x-m_{q}(z)\right)+\overline{B K}\left(m_{\hat{q}}(\hat{x})-m_{q}(z)\right) \\
= & \bar{A}\left(x-m_{q}(z)\right)+\overline{B K}\left(m_{\hat{q}}(\hat{x})-x+x-m_{q}(z)\right) \\
= & (\bar{A}+\overline{B K}) e_{2}+\overline{B K}\left(m_{\hat{q}}(\hat{x})-x\right) e_{2}+\overline{B K M_{\hat{q}}}\left(\hat{x}-m_{\hat{q}}(x)\right) \\
= & (\bar{A}+\overline{B K}) e_{2}+\overline{B K M_{\hat{q}}} e_{1}
\end{aligned}
$$


Thus, using $e:=\left[\begin{array}{ll}e_{1}^{T} & e_{2}^{T}\end{array}\right]^{T}$, the continuous dynamics of $e$ can be written as

$$
\dot{e}=\left[\begin{array}{c|c}
\bar{A}+\bar{L} C & 0 \\
\hline \overline{B K}_{\hat{q}} & \bar{A}+\overline{B K}
\end{array}\right] e .
$$

Define now $W_{o}\left(e_{1}\right):=\left|e_{1}\right| \frac{2}{P_{o}}$ and $W_{c}\left(e_{2}\right):=\left|e_{2}\right| \frac{2}{P_{c}}$. From Assumption 4, we have $\dot{W}_{o}\left(e_{1}\right) \leq\left|e_{1}\right|_{\bar{H}_{o}}^{2} \bar{H}_{o}$ during flows, where the matrix $\bar{H}_{o}=H_{o} \otimes I_{2}$ guarantees $\left(\bar{H}_{o}, \bar{A}+\bar{L} C\right)$ observable. Moreover, from (67b), $W_{0}\left(e_{1}^{+}\right) \leq W_{0}\left(e_{1}\right)$ at jumps. Thus, using the coordinates $\left(e_{1}, \sigma,(x, z, q, \hat{q})\right)$ and Lemma 1 ( $A$ in Lemma 1 is given in this case by $\bar{A}+\bar{L} C)$, we get the function $Y_{o}\left(e_{1}, \sigma\right)$ which satisfies $(i)$-(iii) of Lemma 1. In a similar way, when $e_{1}=0$, we have $\dot{W}_{c}\left(e_{2}\right) \leq\left|e_{2}\right|_{\bar{H}_{c}{ }^{T} \bar{H}_{c}}$ during flows, where $\bar{H}_{c}=H_{c} \otimes I_{2}$ and the pair $\left(\bar{H}_{c}, \bar{A}+\overline{B K}\right)$ is observable, and by (67a), $W_{c}\left(e_{2}^{+}\right) \leq W_{c}\left(e_{2}\right)$ at jumps. So, for the coordinates $\left(e_{2}, \sigma,\left(z, q, \hat{q}, e_{1}\right)\right)$, supposing $e_{1}=0$, by Lemma 1 , we get the function $Y_{c}\left(e_{2}, \sigma\right)$ which satisfies $(i)$ - $(i i i)$ of Lemma 1.

Define now $V(X):=\bar{\rho} Y_{o}\left(e_{1}, \sigma\right)+Y_{c}\left(e_{2}, \sigma\right)$, where $\bar{\rho}>0$ is selected below and $X=(z, x, \hat{x}, q, \hat{q}, \sigma)$. Note that $V$ is positive definite with respect to $\mathcal{A}$. Then, considering the cascade structure in (70), and the properties (i)-(iii) of Lemma 1 satisfied by both $Y_{o}$ and $Y_{c}$, and using a constant $\gamma_{2}>0$ such that $\sqrt{\gamma_{2}}\left|e_{2}\right|\left|e_{1}\right| \geq\left\langle\nabla_{e_{2}} Y_{c}\left(e_{2}, \sigma\right),-\overline{B K M}_{\hat{q}} e_{1}\right\rangle$, for $X \in \overline{\mathcal{C}}$, we have

$$
\begin{aligned}
\dot{V}(X) \leq & -\gamma_{1} \bar{\rho} Y_{o}\left(e_{1}, \sigma\right)-\gamma_{1} Y_{c}\left(e_{2}, \sigma\right)+ \\
& +\sqrt{\gamma_{2}}\left|e_{2}\right|\left|e_{1}\right|, \text { for some } \gamma_{1}>0 \\
\leq & -\gamma_{1} \bar{\rho} Y_{o}\left(e_{1}, \sigma\right)-\gamma_{1} Y_{c}\left(e_{2}, \sigma\right)+\frac{\gamma_{2} \gamma_{3}}{2}\left|e_{1}\right|^{2}+ \\
& +\frac{\gamma_{2}}{2 \gamma_{3}}\left|e_{2}\right|^{2}, \text { for any } \gamma_{3}>0 \\
\leq & -\gamma_{1} \bar{\rho} Y_{o}\left(e_{1}, \sigma\right)-\gamma_{1} Y_{c}\left(e_{2}, \sigma\right)+\frac{\gamma_{2} \gamma_{3} \gamma_{4}}{2} Y_{o}\left(e_{1}, \sigma\right)+ \\
& +\frac{\gamma_{2} \gamma_{4}}{2 \gamma_{3}} Y_{c}\left(e_{2}, \sigma\right), \text { for some } \gamma_{4}>0 \\
= & -\frac{\gamma_{1}}{2}\left(\bar{\rho} Y_{o}\left(e_{1}, \sigma\right)+Y_{c}\left(e_{2}, \sigma\right)\right) \\
= & -\frac{\gamma_{1}}{2} V(X)
\end{aligned}
$$

where $\gamma_{3}:=\frac{\gamma_{2} \gamma_{4}}{\gamma_{1}}$ and $\bar{\rho}:=\frac{\gamma_{2} \gamma_{3} \gamma_{4}}{\gamma_{1}}$. Moreover, from (67) and Lemma 1, for each $X \in \overline{\mathcal{D}}$ we have

$$
V\left(X^{+}\right)=\bar{\rho} \gamma Y_{o}\left(e_{1}, \sigma\right)+\gamma Y_{c}\left(e_{2}, \sigma\right)=\gamma V(X),
$$

for some $\gamma \in[0,1)$. Then, using the bounds in Proposition 9, by [29, Theorem 1] the set $\mathcal{A}$ is GES.

Remark 14: Sufficient conditions for Theorem 6 are presented in Section V-C. Intuitively, the input $u_{c}$ and the definition of $\delta$ parallel the tracking case of Section $\mathrm{V}$, where $u_{c}$ in (57a) differs from $u$ in (40a) only for the term $x$ replaced by $m_{\hat{q}}(x)$. In a similar way, the input $u_{o}$ and $\delta$ parallel the observer case of Section V, where $u_{o}$ in (57b) differs from $u$ in (40b) for the new term $u_{c}$.

Example 2: Consider a translating mass $\mathcal{X}$ on a rectangular billiard (Proposition 7) which tracks the reference $\mathcal{Z}$. The billiard is represented in Figure 6 and is defined by $s_{\circ}=0$ and

$$
\left[F_{1}\left|F_{2}\right| F_{3} \mid F_{4}\right]:=\frac{1}{4}\left[\begin{array}{l|l|l|l}
3 & 0 & -3 & 0 \\
0 & 4 & 0 & -4
\end{array}\right] .
$$

Using (38) and Proposition 7, the dynamics of the closed loop system is given by equations (56)-(60),

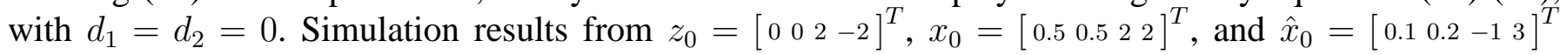
are reported in Figure 7, respectively for $\hat{q}_{0}=q_{0}=0$, and $q_{0}=3, \hat{q}_{0}=8$ and clearly illustrate the asymptotic tracking properties established in Theorem 6. 

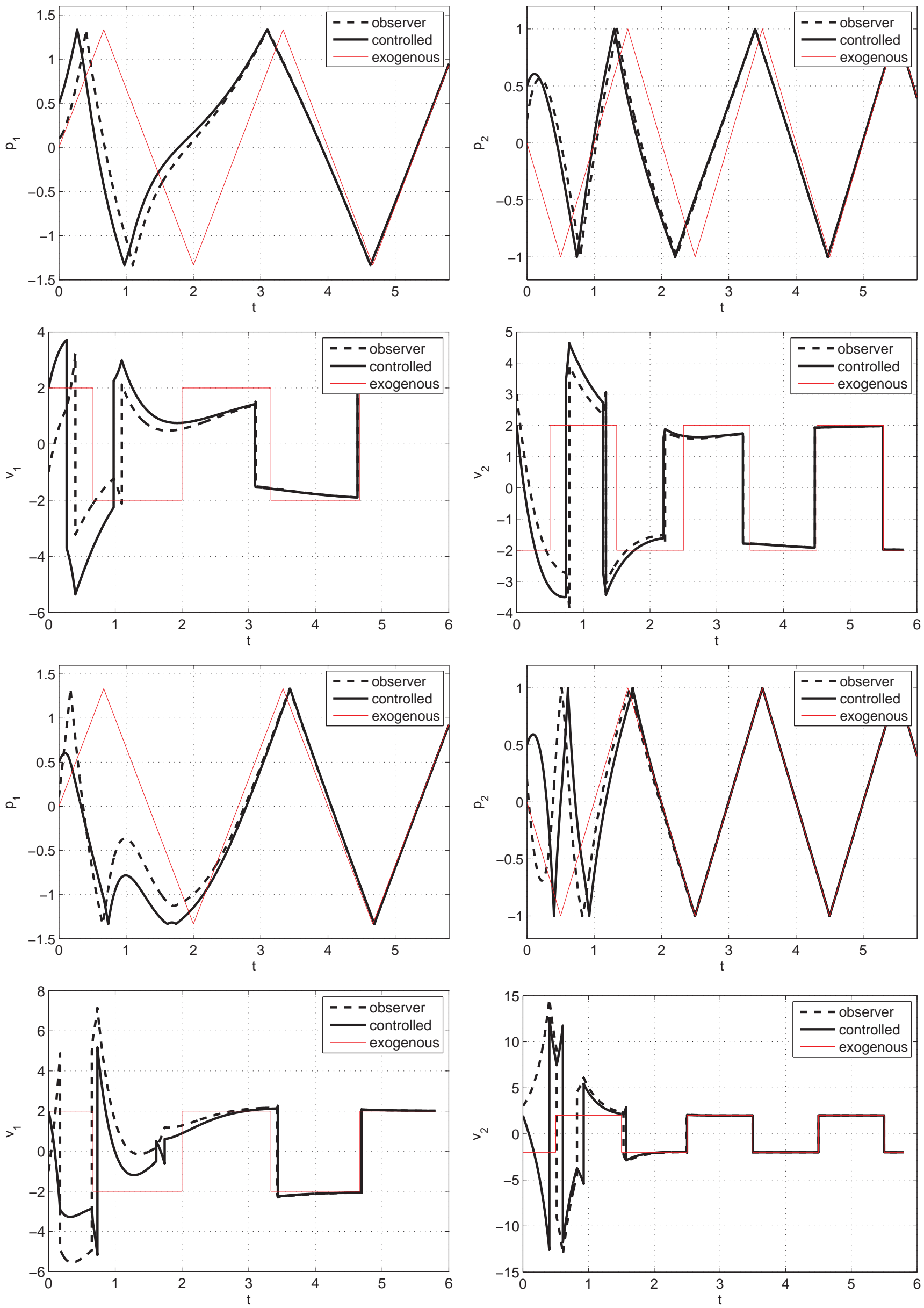

Figure 7. Example 2. First row: positions, $q_{0}=\hat{q}_{0}=0$. Second row: velocities, $q_{0}=\hat{q}_{0}=0$. Third row: positions, $q_{0}=3, \hat{q}_{0}=8$. Fourth row: velocities, $q_{0}=3, \hat{q}_{0}=8$. 


\section{CONCLUSION}

We introduced a hybrid model for the impact dynamics of two translating masses within billiards and we proposed two algorithms which guarantee global exponential tracking and global exponential state estimation for billiards whose shape is defined by a single wall, two parallel walls, two perpendicular walls, and rectangles. Then, by combining these two algorithms, we achieved global exponential tracking by output feedback. Each algorithm presented is robust to impact detection delays and does not require high gain feedback.

\section{REFERENCES}

[1] J.M. Bourgeot and B. Brogliato. Tracking control of complementarity Lagrangian systems. Int. J. Bifurcation Chaos, 15(6):1839-1866, 2005.

[2] B. Brogliato. Nonsmooth mechanics: models, dynamics, and control. Springer Verlag, 1999.

[3] B. Brogliato. Absolute stability and the Lagrange-Dirichlet theorem with monotone multivalued mappings. Systems \& control letters, 51(5):343-353, 2004.

[4] B. Brogliato, S. I. Niculescu, and P. Orthant. On the control of finite-dimensional mechanical systems with unilateral constraints. IEEE Transactions on Automatic Control, 42(2):200-215, 1997.

[5] M. Buhler, D.E. Koditschek, and P.J. Kindlmann. A family of robot control strategies for intermittent dynamical environments. IEEE Control Systems Magazine, 10(2):16-22, 1990.

[6] C. Cai, A.R. Teel, and R. Goebel. Smooth Lyapunov functions for hybrid systems Part II:(pre) asymptotically stable compact sets. IEEE Transactions on Automatic Control, 53(3):734-748, 2008.

[7] F. Forni, A.R. Teel, and L. Zaccarian. Tracking control in billiards using mirrors without smoke, part I: Lyapunov-based local tracking in polyhedral regions. In Joint CDC-ECC, pages 3283-3288, Orlando (FL), USA, December 2011.

[8] F. Forni, A.R. Teel, and L. Zaccarian. Tracking control in billiards using mirrors without smoke, part II: additional Lyapunov-based local and global results. In Joint CDC-ECC, pages 3289-3294, Orlando (FL), USA, December 2011.

[9] S. Galeani, L. Menini, A. Potini, and A. Tornambè. Trajectory tracking for a particle in elliptical billiards. Int. J. of Control, 81(2):189-213, February 2008.

[10] S. Galeani, L. Menini, and A. Tornambe. A local observer for linearly observable nonlinear mechanical systems subject to impacts. In Proceedings of the 2003 American Control Conference., volume 6, pages 4760 - 4765 vol.6, June 2003.

[11] R. Goebel, R. Sanfelice, and A.R. Teel. Hybrid dynamical systems. IEEE Control Systems Magazine, 29(2):28-93, April 2009.

[12] R. Goebel and A.R. Teel. Solutions to hybrid inclusions via set and graphical convergence with stability theory applications. Automatica, 42(4):573 - 587, 2006.

[13] W. Heemels and B. Brogliato. The complementarity class of hybrid dynamical systems. European Journal of Control, 9(2-3):322-360, 2003.

[14] R.I. Leine and N. van de Wouw. Stability and convergence of mechanical systems with unilateral constraints. Lecture Notes in Applied and Computational Mechanics. Springer Verlag, 2008.

[15] R.I. Leine and N. van de Wouw. Uniform convergence of monotone measure differential inclusions: with application to the control of mechanical systems with unilateral constraints. International Journal of Bifurcation and Chaos in Applied Sciences and Engineering, 18(5):1435-1457, 2008.

[16] L. Menini and A. Tornambè. Asymptotic Tracking of Periodic Trajectories for a Simple Mechanical System Subject to Nonsmooth Impacts. IEEE Trans. Aut. Control, 46:1122-1126, 2001.

[17] L. Menini and A. Tornambè. Dynamic position feedback stabilization of multi-degrees-of-freedom linear mechanical systems subject to non-smooth impacts. IEE Proceedings of Control Theory and Applications, 148(6):488-496, November 2001.

[18] L. Menini and A. Tornambè. Velocity observers for linear mechanical systems subject to single non-smooth impacts. Systems \& Control Letters, 43:193-202, 2001.

[19] I.C. Morarescu and B. Brogliato. Trajectory tracking control of multiconstraint complementarity Lagrangian systems. IEEE Transactions on Automatic Control, 55(6):1300-1313, 2010.

[20] B. Morris and J.W. Grizzle. Hybrid invariant manifolds in systems with impulse effects with application to periodic locomotion in bipedal robots. IEEE Trans. Aut. Cont., 54(8):1751-1764, 2009.

[21] PR Pagilla. Control of contact problem in constrained Euler-Lagrange systems. IEEE Trans. Aut. Cont., 46(10):1595-1599, 2001.

[22] PR Pagilla and B. Yu. An experimental study of planar impact of a robot manipulator. IEEE/ASME Transactions on Mechatronics, 9(1):123-128, 2004.

[23] R. Ronsse, P. Lefèvre, and R. Sepulchre. Sensorless stabilization of bounce juggling. IEEE Trans. on Robotics, 22(1):147-159, 2006.

[24] R.G. Sanfelice. Robust Hybrid Control Systems. PhD thesis, University of California, Santa Barbara, 2007.

[25] R.G. Sanfelice, R. Goebel, and A.R. Teel. Invariance principles for hybrid systems with connections to detectability and asymptotic stability. IEEE Transactions on Automatic Control, 52(12):2282-2297, 2007.

[26] R.G. Sanfelice, R. Goebel, and A.R. Teel. Generalized solutions to hybrid dynamical systems. ESAIM: COCV, 14(4):699-724, October 2008.

[27] W.J. Schwind and D.E. Koditschek. Control of forward velocity for a simplified planar hopping robot. In IEEE International Conference on Robotics and Automation, volume 1, pages 691-696, 1995.

[28] P. Sekhavat, N. Sepehri, and Q. Wu. Impact stabilizing controller for hydraulic actuators with friction: Theory and experiments. Control Engineering Practice, 14(12):1423-1433, 2006. 
[29] A.R. Teel, F. Forni, and L. Zaccarian. Lyapunov-based sufficient conditions for exponential stability in hybrid systems. IEEE Trans. Aut. Cont., 58(6):1591-1596, 2013.

[30] A. Tornambe. Modeling and control of impact in mechanical systems: Theory and experimental results. IEEE Trans. Aut. Cont., 44(2):294-309, 1999. 